\title{
Cheniella gen. nov. (Leguminosae: Cercidoideae) from southern China, Indochina and Malesia
}

\author{
Ruth P. CLARK ${ }^{1, *}$, Barbara A. MACKINDER ${ }^{1,2}$ \& Hannah BANKS ${ }^{3}$ \\ ${ }^{1,3}$ Herbarium, Royal Botanic Gardens, Kew, Richmond, Surrey, TW9 3AE, UK. \\ ${ }^{2}$ Royal Botanic Garden, Edinburgh, 20A Inverleith Row, EH3 5LR, UK. \\ *Corresponding author: R.Clark@kew.org \\ ${ }^{2}$ Email: B.Mackinder@,rbge.ac.uk \\ ${ }^{3}$ Email: $\underline{\text { H.banks@kew.org }}$
}

\begin{abstract}
For much of the last thirty years, the caesalpinioid genus Bauhinia has been recognised by numerous authors as a broadly circumscribed, ecologically, morphologically and palynologically diverse pantropical taxon, comprising several subgenera. One of these, Bauhinia subg. Phanera has recently been reinstated at generic rank based on a synthesis of morphological and molecular data. Nevertheless, there remains considerable diversity within Phanera. Following a review of palynological and molecular studies of Phanera in conjunction with a careful re-examination of the morphological heterogeneity within the genus, we have found strong evidence that the species of Phanera subsect. Corymbosae are a natural group that warrant generic status. We describe here the genus Cheniella R.Clark \& Mackinder gen. nov. to accommodate them. It comprises 10 species and 3 subspecies, one newly described here. Generic characters include leaves that are simple and emarginate or bilobed; flowers with elongate hypanthia which are as long as or much longer than the sepals; pods that are glabrous, compressed, oblong, indehiscent or tardily dehiscent; and with numerous seeds, the seeds bearing an unusually long funicle extending most of the way around their circumference. A further distinctive floral character was found to be a fleshy disc on which the staminodes are mounted. An analysis carried out for this study reveals Cheniella to be characterised by a pollen type that is unique to the genus and previously unknown in the Leguminosae. Species diversity is richest in southern China, the full distribution extending westward to India and south- and eastward through Indochina into Malesia.
\end{abstract}

Keywords. Bauhinia, Caesalpinioideae, Cercideae, Fabaceae, Phanera, pollen, staminodes.

Clark R.P., Mackinder B.A. \& Banks H. 2017. Cheniella gen. nov. (Leguminosae: Cercidoideae) from southern China, Indochina and Malesia. European Journal of Taxonomy 360: 1-37. https://doi.org/10.5852/ejt.2017.360

\section{Introduction}

The legume family (Leguminosae Juss.), with about 19,500 species (LPWG 2013), is the third most species rich family of the angiosperms after the orchid family (Orchidaceae Juss., ca 25,000-30,000 species (Wilkin \& Schuiteman 2015)) and the daisy family (Compositae Giseke, ca 25,000 species (Hind 2015)). Traditionally, the Caesalpinoideae DC., was one of three subfamilies recognised in the Leguminosae, containing ca 2,300 species in 171 genera, arranged in 4 tribes including the Cercideae 
Bronn. Following the recent major rearrangement of the family into six subfamilies, the Caesalpinioideae now comprises 148 genera with ca 4,400 species, whilst tribe Cercideae is elevated to subfamily rank as the Cercidoideae LPWG, with 12 genera and. ca 335 species (LPWG 2017).

This study concerns the Cercidoideae, putatively the most basally branching extant subfamily of Leguminosae (LPWG 2013, 2017), which, as the Cercideae, was further subdivided into the subtribes Cercidinae Wunderlin and Bauhiniinae Wunderlin (Wunderlin 1979; Wunderlin et al. 1981). Cercidinae (sensu Wunderlin 1979) comprised only three, long-recognised genera, Cercis L., Adenolobus (Harv. ex Benth. \& Hook.f.) Torre. \& Hillc. and Griffonia Baill. In contrast, within the subtribe Bauhiniinae (sensu Wunderlin 1979), generic concepts have been less stable. Over a five-year period, the number of genera recognised in this subtribe varied from 26 genera (Wunderlin 1976) to only one, Bauhinia L. s. lat. (Wunderlin et al. 1981). Since 1981, the authors of regional taxonomic accounts have commonly upheld the concept of a single genus, a broadly circumscribed, morphologically and palynologically diverse Bauhinia s. lat., comprising ca 300-350 spp. (Larsen et al. 1980, 1984; Hou et al. 1996; Dezhao et al. 2010). However, molecular based analyses have provided evidence that Bauhinia s. lat. is not a natural taxon (Bruneau et al. 2001; Hao et al. 2003; Bruneau et al. 2008; Sinou et al. 2009). The proposal of Lewis \& Forest (2005) to recognise Bauhinia s. str. and eight generic segregates, Barklya F.Muell., Brenierea Humbert, Gigasiphon Drake, Lasiobema (Korth.) Miq., Lysiphyllum (Benth.) de Wit, Phanera Lour., Piliostigma Hochst. and Tylosema (Schweinf.) Torre \& Hillc. was largely supported by the findings of Sinou et al. (2009) in the most broadly sampled published phylogenetic study of the tribe Cercidoideae (then tribe Cercideae) to date. Soon after, Wunderlin (2010) formally reorganised the Cercidoideae (as the Cercideae) into 12 genera. In addition, a widening acceptance of the reclassification of Bauhinia s. lat. as Bauhinia s. str. and distinct segregate genera is evident from the recognition in recent publications of Phanera (de Queiroz 2006; Vaz 2010; Wunderlin 2011; Bandyopadhyay et al. 2012; Kumar et al. 2014; Mackinder \& Clark 2014), Lysiphyllum (Bandyopadhyay \& Ghoshal 2014) and Schnella Raddi (Wunderlin 2011; Trethowan et al. 2015) as distinct.

The genus Phanera is morphologically distinct from Bauhinia s. str.: the species of Phanera are tendrilled lianas with (2-)3 stamens per flower, whereas Bauhinia s. str. are trees with 1-10 stamens per flower (de Wit 1956; Verdcourt 1979). Further evidence to support the segregation of the genus is provided by studies on flavonoid data (Salatino et al. 1999), intron presence data (Lai et al. 1997) and volatile oil data (Duarte-Almeida et al. 2004).

\section{Phanera circumscription and relationships}

Until recently, Phanera was considered to comprise 120-130 lianescent species from southeast Asia and South America (Lewis \& Forest 2005). However, following the segregation of the neotropical species as the genus Schnella (Trethowan et al. 2015), Phanera was effectively recircumscribed as a group of ca 90 species, restricted to southeast Asia. This genus, which is most diverse in Malesia, remains the largest of the segregate genera reinstated from Bauhinia s. lat. (Mackinder \& Clark 2014). Nevertheless, Phanera is still a somewhat heterogeneous taxon. In particular, as several authors have already noted, the generic boundary of Phanera with regard to Lasiobema warrants more detailed research (Hao et al. 2003; Lewis \& Forest 2005; Mackinder \& Clark 2014; Trethowan et al. 2015). Sinou et al. (2009) indicated, albeit with weak support, that based on a phylogenetic analysis of nucleotide sequence data from the cpDNA trnL-F region, Asian Phanera are closely related to Lasiobema; although sampling in that study was very limited. Both the close relationship between Phanera and Lasiobema, as well as the wider infrageneric phylogenetic framework of Cercidoideae (the then Cercideae) resolved by Sinou et al. (2009), were congruent with the findings of Hao et al. (2003) in a phylogenetic study of the Cercidoideae (as the Cercideae) based on nucleotide data from the Internal Transcribed Spacer (ITS) region. The phylogenetic structure of the main lineages as determined by those two studies is compared (Fig. 1). In Hao et al. (2003), the Schnella lineage was represented by an accession of Bauhinia glabra 
Jacq. now recognised as Schnella glabra (Jacq.) Dugand. In Sinou et al. (2009), several accessions of Schnella were sampled and placed together on a lineage labelled as American Phanera in Fig. 1 of that publication.

The study of Hao et al. (2003), designed to investigate the close relationship between SE Asian Phanera and Lasiobema, recovered a strongly supported clade containing 31 accessions, representing 27 species, of which 23 were species of Phanera and four were species of Lasiobema. Neither genus was resolved as individually monophyletic. A plausible evolutionary interpretation of these findings is that neither Phanera nor Lasiobema are natural groups as currently delimited and that, given the morphological diversity that still exists within both genera, a further division of both genera is likely to be necessary if we are to achieve a classification that reflects monophyly. Indeed, Wunderlin et al. (1987) proposed

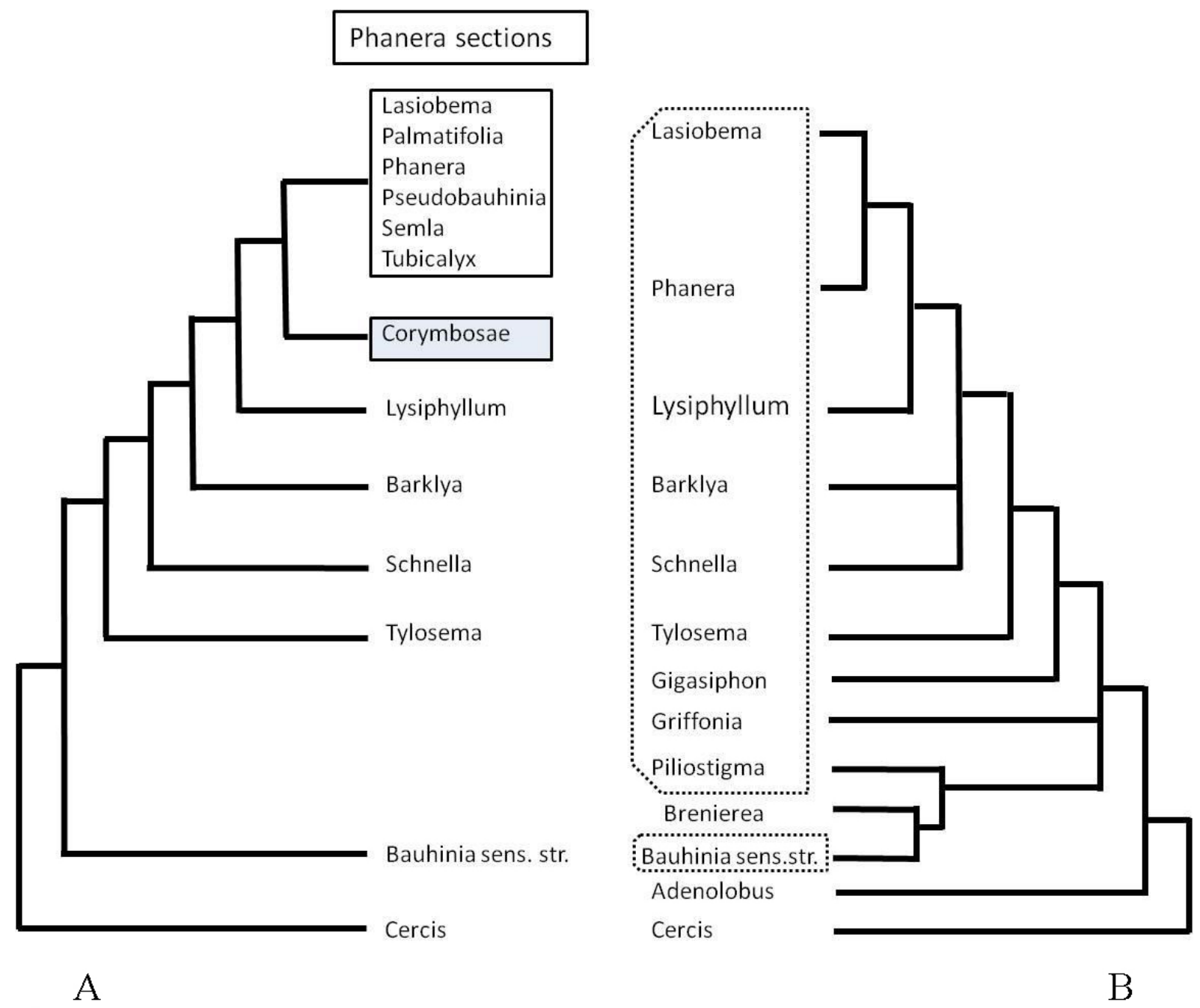

Fig. 1. Comparative topologies of two separate phylogenetic analyses of tribe Cercideae Bronn. A. Topology of the parsimony strict consensus tree based on the nrDNA Internal Transcribed Spacer region (Hao et al. 2003). B. Bayesian majority rule tree topology derived from an analysis of data from the cpDNA trnL-F region (Sinou et al. 2009). Note that the reinstatement of the genus Schnella (Wunderlin 2010) post-dates both phylogenetic studies. 
that the Asian species of Phanera (treated by him in that publication under Bauhinia subg. Phanera) should be further divided among seven sections, and that the species of Lasiobema be placed in three further sections. Larsen (1975) demonstrated two different pollen types in Lasiobema which supported the hypothesis of de Wit (1956) that Lasiobema may be better recognized as two genera based on floral morphology.

Nonetheless, within Phanera s. lat., the species of Phanera sect. Corymbosae de Wit do form a morphologically homogeneous group that is readily identifiable as distinct from all other Phanera diversity. Five species of Phanera sect. Corymbosae were sampled by Hao et al. (2003) and were resolved as a strongly supported (100\% bootstrap) monophyletic group and placed as sister to a clade containing all other accessions of Phanera and Lasiobema species. Here we present the case for the recognition of Phanera sect. Corymbosae as a genus in its own right.

\section{Material and methods}

The synoptical descriptions, the key to species, and distribution data were created using a combination of the existing literature, herbarium specimens at BM, E and K, and those available online via JSTOR (http:// plants.jstor.org) and the Chinese Virtual Herbarium (http://www.cvh.ac.cn/). Preliminary conservation assessments were made using the categories and criteria of IUCN (2001). Locality data were derived from specimens from the herbaria listed above as well as specimens detailed in the Flore du Camboge, Laos, et Vietnam (Larsen et al. 1980). It was not possible to use locality data from the Flora of China for the assessments, as only occurrence by province is recorded; thus the Extent of Occurrence (EOO) reported in this account may in some cases be smaller than the true value, which in turn may have the effect of slightly inflating the category of threat.

The systematic arrangement of the species was created by inferring relationships based on morphological similarity, and on geographical distribution.

For pollen studies, mature buds were obtained from herbarium specimens held at the Royal Botanic Gardens, Kew (K) (Appendix 1). These were dissected in a 1\% solution of nonionic wetting agent using a Leica Wild M8 microscope. Pollen was washed and then acetolysed (Erdtman 1960). The pollen grains were prepared for examination by scanning electron microscope (SEM) by pipetting acetolysed pollen exines in $95 \%$ ethanol onto specimen stubs. These were left to air dry before being sputter-coated with platinum using a Quorum 150TES sputter coater. They were examined using a Hitachi S-4700 II field emission SEM. The pollen terminology follows the glossary published by Punt et al. (2007).

Of the samples used for the floral anatomy study with SEM, specimen Owens s.n. was stored in Kew Mix (5\% formaldehyde, $5 \%$ glycerol, $37 \%$ water, $53 \%$ industrial methylated spirit), and flowers of Nichols 1292 were taken from dried herbarium specimens and rehydrated in boiling water for 10 minutes. Specimens Clark 418, 426 and 458 were collected into and preserved in $70 \%$ ethanol. For scanning electron microscopy (SEM), flowers and floral buds were dissected in $70 \%$ ethanol, dehydrated to $100 \%$ ethanol, and critical point dried using an Autosamdri-815B critical-point dryer. Dried samples were mounted onto aluminum stubs and coated with platinum in a Quorum Q150T sputter coater. SEM images were taken with a Hitachi S-4700-II cold field emission SEM.

Secondary veins as defined in this manuscript are the leaf veins arising from the leaf base, and approximately parallel to the primary central vein. Measurements in square brackets are those derived from literature, not observed by the present authors. 


\section{Results}

\section{Proposal to recognise Phanera sect. Corymbosae as a distinct genus}

In his detailed account of the (then) 43 Malesian species of Phanera, de Wit (1956) first delimited Phanera sect. Corymbosae to accommodate Phanera corymbosa Benth. (type) and Phanera glauca Benth. These two species have narrow, elongate hypanthia, the length of which greatly exceeds the length of the sepals, in contrast to all other species from the region, which have a hypanthium that is in most cases much shorter than, or occasionally equal to the sepals. De Wit (1956) also observed that the compressed fruits of Phanera corymbosa and Phanera glauca contained numerous seeds, many more than other species from the region. Both Phanera corymbosa and Phanera glauca have glabrous ovaries, a character that is unusual among species of Phanera. In 1988, T.C. Chen described three new legume species from China (in Bauhinia s. lat.) as Bauhinia ovatifolia T.C.Chen, Bauhinia damiaoshanensis T.C.Chen and Bauhinia quinnanensis T.C.Chen which also possess the combination of elongate hypanthia and a glabrous or subglabrous ovary (Chen 1988).

In a cladistic analysis of Bauhinia, Zhang \& Chen (1992) expanded the Corymbosae group, which they defined as a series within Bauhinia, to include the three recently described species of Chen (1988), and additionally Bauhinia clemensiorum Merr., Bauhinia didyma H.Y.Chen, Bauhinia lakhonensis Gagnep. and Bauhinia touranensis Gagnep. We have found that all species included in Bauhinia ser. Corymbosae as defined by Zhang \& Chen (1992) exhibit the elongate hypanthium, glabrous or subglabrous ovary, and the long, glabrous, indehiscent (or tardily dehiscent), many-seeded fruits that characteristic of the section.

In his description, de Wit (1956) described the seeds of the two original species as having a funicle that extends as a rim along more than half the circumference of the seed, a funicle type unknown in other species of Phanera. We have also seen seeds of Bauhinia clemensiorum, Bauhinia quinnanensis and Bauhinia tenuiflora Watt ex C.B.Clarke and confirm that they too have this unusual type of funicle. Flowers of the Corymbosae contain seven staminodes, of which five are mounted on a fleshy disc, a structure that appears to be unique within the context of Phanera s. lat. Zhang (1994) concluded Bauhinia sect. Corymbosae to be a natural group based on his study of leaf venation in Cercidoideae (the then Cercideae), describing the Corymbosae leaf as having "primary veins not marginal, the marginal ultimate venation looped, well developed areoles, and simple veinlets".

In this study, we raise the status of Bauhinia glauca subsp. tenuiflora (Watt ex C.B.Clarke) K.Larsen \& S.S.Larsen to the rank of species, bringing to 10 the total number of species which we consider to belong to Phanera sect. Corymbosae of de Wit (1956). Together, the 10 species of sect. Corymbosae form a morphologically homogeneous group that can be clearly distinguished from the rest of Phanera.

Pollen morphology in legumes has frequently been demonstrated to be taxonomically informative at the genus level, and overviews of the great diversity of pollen morphology found in subfam. Caesalpinioideae (as then circumscribed) have previously been documented by Graham \& Barker (1981), and Banks (Banks \& Klitgaard 2000; Banks et al. 2003; Banks et al. 2006). The overview of pollen in the subfam. Caesalpinioideae (former circumscription) shows that the greatest diversity in pollen structure occurs in the earlier branching taxa, while the taxa of later diverging lineages appear to have more constrained pollen morphology (Banks et al. 2003). The pollen of Bauhinia s. lat. is known to exhibit a great range of morphological features, in terms of ornamentation, apertures, and units of dispersal (dos Santos et al. 2012).

The monophyletic Cercidoideae is one of the earliest branching lineages in Leguminosae, sister to the rest of the family (LPWG 2013, 2017). The pollen of the Cercidoideae has long been known to be 
diverse and varied, but problematic species and generic delimitations have previously hindered our understanding of the similarities, differences, evolution and taxonomic significance of the various pollen structures found within the tribe, until the studies of Banks et al. $(2013,2014)$.

Previous published studies of the pollen of Bauhinia s. lat. have mostly been restricted to regional accounts (Larsen 1975; Larsen \& Larsen 1982, 1991, 1993; Gamerro \& Fortunato 2001) although Ferguson \& Pearce (1986) carried out a wider survey of neotropical species. Ferguson \& Pearce (1986) described two patterns of morphological variation in pollen and found that apertures and exine stratification correlated with taxonomy, whereas ornamentation, pollen size and exine thickness showed a great deal of continuous variation and also examples of homoplasy. In many cases homoplasious morphological variation is correlated with floral morphology and pollination syndromes (e.g., supratectal gemmae and verrucae appeared to be correlated with bat pollination) (Ferguson \& Pearce 1986) and has evolved iteratively in the tribes Cercideae and Detarieae (Banks \& Rudall 2016).

Recent studies in the phylogenetics of Bauhinia s. lat. allowed us to re-evaluate pollen structures in this group (Banks et al. 2013, 2014) and assess the morphological variation using a molecular based phylogenetic framework. These studies identified pollen characters of potential taxonomic importance and assessed the evolution of pollen structure by examining the variation of pollen types in Bauhinia s. lat. within the phylogenetic context provided by the study of Sinou et al. (2009). The pollen of Cheniella R.Clark \& Mackinder gen. nov. is revealed to have the structure first described by Larsen (1975) as the "Glauca type". Cheniella gen. nov. pollen is isopolar, tricolporate, with apertures usually syncolporate (Fig. 2A-E), and is oblate spheroidal to oblate in shape (Fig. 2A, C-E). Ornamentation is coarsely rugulate to verrucate (Fig. 2F), with larger verrucae along the aperture margins (Fig. 2B) and sometimes anastomosing at aperture margins, forming a defined margin to the apertures. Aperture membranes are verrucate, with coarser verrucae over the pores forming operculae (Fig. 2A-E).

The palynological investigation carried out as part of this study indicates that the species of the sect. Corymbosae (four species sampled, of a total of nine at the time of study) share a pollen morphology that is unique within Bauhinia, and within the Cercidoideae. No other pollen in the Leguminosae is known to have the combination of syncolporate apertures and verrucate ornamentation (Fig. 2B). However, similar types of verrucate ornamentation have previously been seen in taxa pollinated by butterflies, moths, birds or bats (Banks \& Rudall 2016).

Hao et al. (2003) constructed a well resolved phylogeny of the subtribe Bauhiniinae (sensu Wunderlin 1979) in which 7 accessions were sampled that represented 5 species from sect. Corymbosae, including the type. The 7 accessions formed a strongly supported (bootstrap $=100 \%$ ) monophyletic group placed as sister (bootstrap $=89 \%$ ) to all other accessions sampled from Phanera and Lasiobema (Fig. 1).

The centre of diversity of sect. Corymbosae is southern China and northern Indo-China, the distribution being principally more northern and more seasonal than that of the majority of Phanera and Lasiobema, which extend into regions that are more tropical, lacking a dry season (Fig. 3).

We consider there is sufficient morphological, palynological, molecular, and geographical evidence to justify the recognition of sect. Corymbosae at generic rank and we hereby describe a new genus, Cheniella gen. nov. 


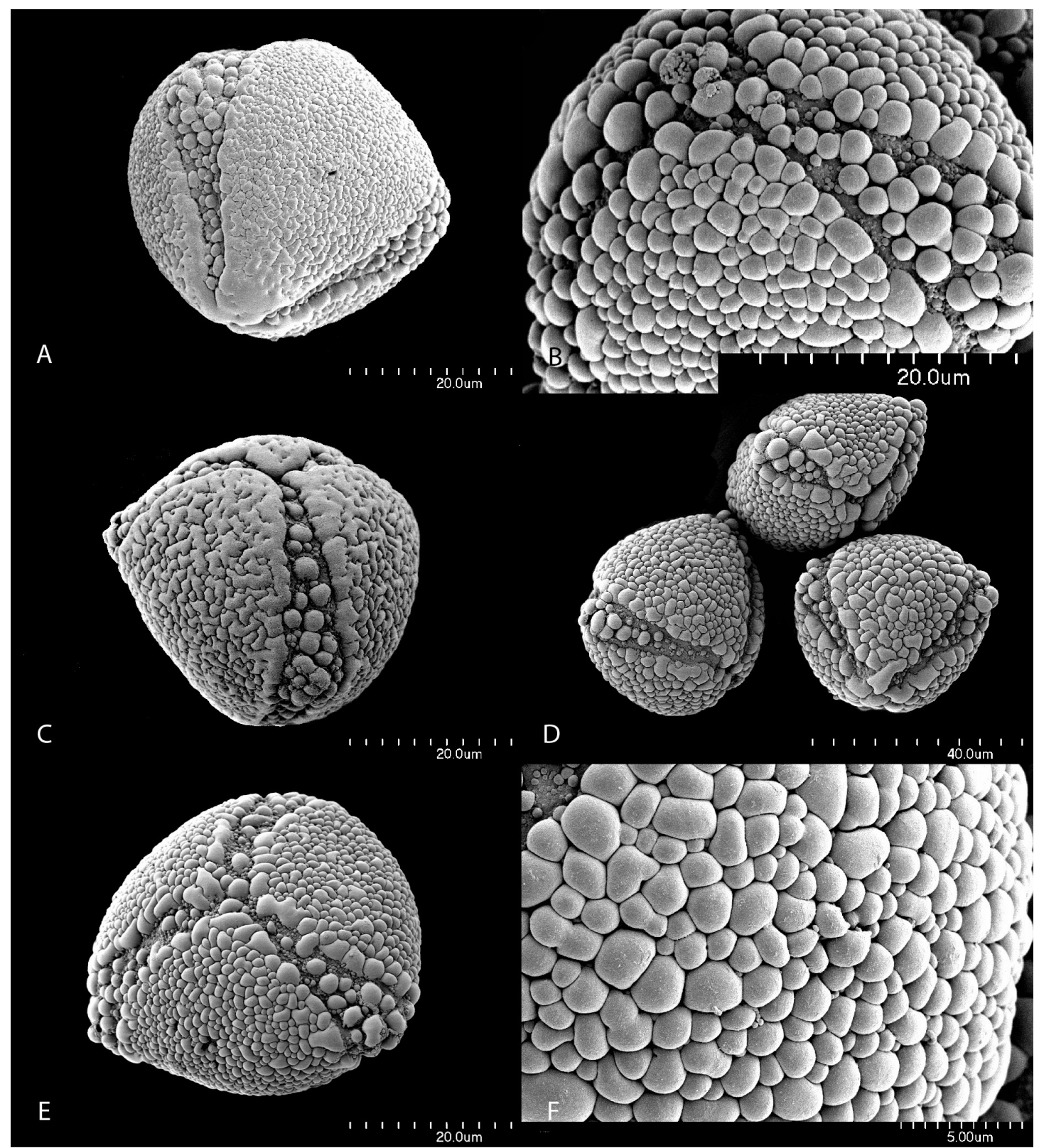

Fig. 2. Pollen. A. Cheniella clemensiorum (Merr.) R.Clark \& Mackinder comb. nov., whole grain in oblique equatorial view showing mesocolpial area. B. C. touranensis (Gagnep.) R.Clark \& Mackinder comb. nov., close up of polar view showing syncolporate apertures and aperture margins defined by larger verrucae. C. C. damiaoshanensis (T.C.Chen) R.Clark \& Mackinder comb. nov., whole grain in oblique equatorial view showing aperture area, with aperture margins defined by larger and anastomosing verrucae, coarsely verrucate aperture membrane, and syncolporate polar area. D. C. touranensis (Gagnep.) R.Clark \& Mackinder comb. nov., group of three grains in different orientations showing their shape and an overview of apertures and ornamentation. E. C. corymbosa (Roxb.) R.Clark \& Mackinder comb. nov., whole grain in polar view showing syncolporate apertures. F. C. touranensis (Gagnep.) R.Clark $\&$ Mackinder comb. nov., close up of mesocolpial area showing surface ornamentation. 


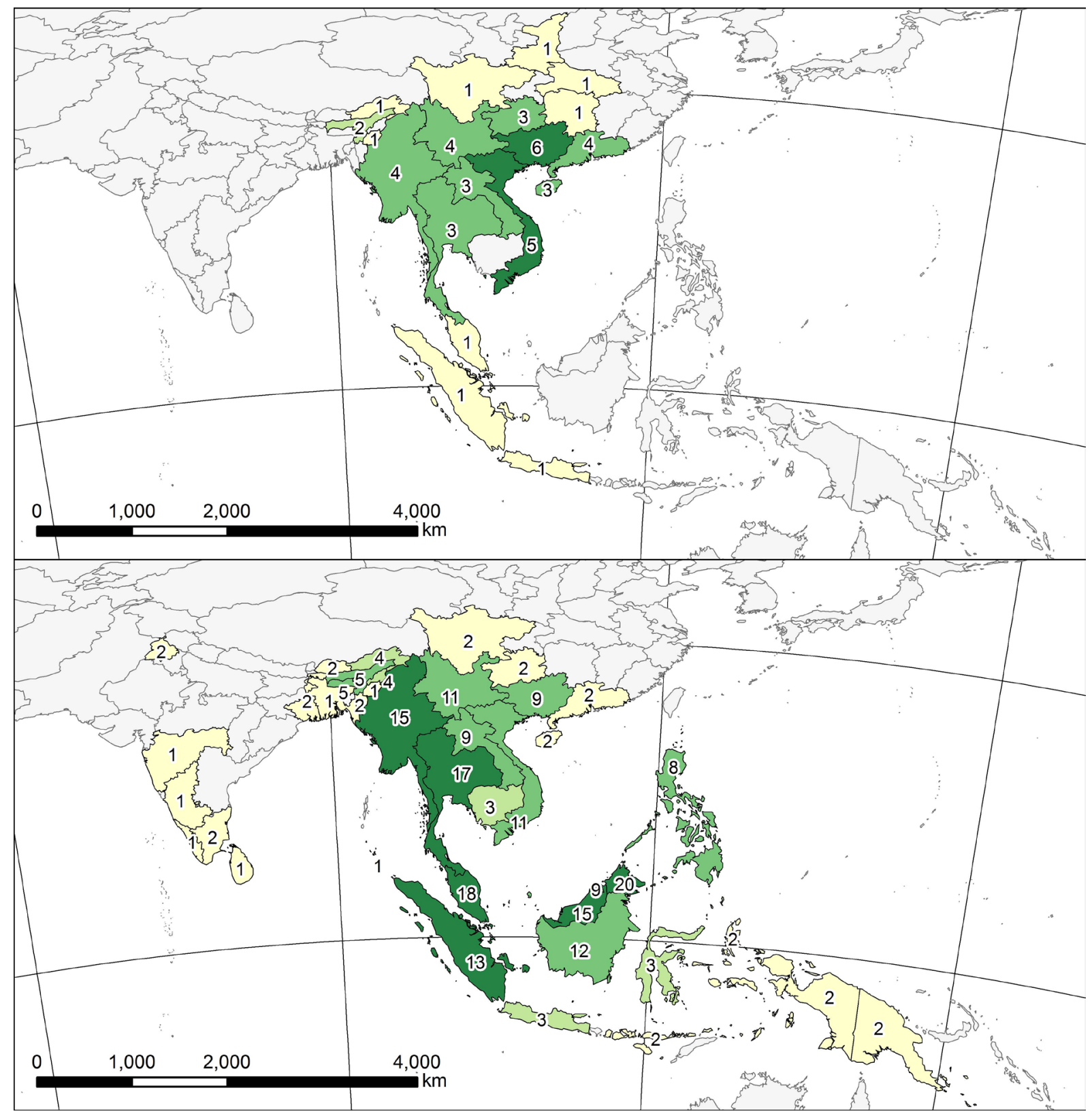

Fig. 3. Relative species density. A. Cheniella R.Clark \& Mackinder gen. nov. B. Phanera Lour. 


\author{
Class Magnoliopsida Brongn. \\ Order Magnoliales Juss. ex Bercht. \& J.Presl \\ Family Leguminosae Juss. \\ Subfamily Cercidoideae LPWG
}

Cheniella R.Clark \& Mackinder gen. nov.
urn:lsid:ipni.org:names:77165904-1

Fig. 4

Phanera subg. Phanera subsect. Corymbosae de Wit, Reinwardtia 3 (4): 387 (de Wit 1956). - Bauhinia sect. Phanera ser. Corymbosae (de Wit) Wunderlin, K. Larsen \& S.S. Larsen, Biologiske skrifter 28: 19 (Wunderlin et al. 1987).

Bauhinia ser. Corymbosae Zhang \& T.C.Chen, Acta Botanica Austro Sinica 8: 31 (Zhang \& Chen 1992).

\title{
Diagnosis
}

Similar to Phanera, but with an elongate hypanthium, equal in length or much longer than the sepals (vs hypanthium shorter or absent), fruit indehiscent (vs usually dehiscent), fruit many-seeded (10-)15$30(-36)$ seeds (not (1-)3-7(-12) seeds), funicle extending most of the circumference of the seed (vs funicle much shorter).

\section{Etymology}

Named for Prof. Chen Dezhao (Chen Te-chao), who described three of the (then) five species of Cheniella gen. nov. known from China. The fossil species Bauhinia cheniae Qi Wang, Z.Q.Song, Y.F.Chen, S.Shen \& Z.Y.Li (Wang et al. 2014) from the late Eocene-Oligocene Ningming Formation in Ningming County (Guangxi Zhuang Autonomous Region, South China) was likewise named for Prof. Chen Dezhao.

\section{Type}

Bauhinia corymbosa Roxb., Flora Indica 2: 329 (Roxburgh 1832).

\section{Genus description}

Lianas or scandent shrubs with tendrils. Leaves entire, emarginate, or bilobed; secondary veins 4-10, tertiary venation highly reticulate. Inflorescence corymbose or racemose. Bracts at base of axis, linear. Bracteoles in pairs midway along the pedicel, filiform. Hypanthium elongate, at least as long as but usually much longer than the sepals, longitudinally ridged. Calyx 5-lobed, splitting irregularly into 2-5 lobes at anthesis. Petals 5, subequal to unequal, short-clawed, hairy on the outer surface; usually white, otherwise pink, or yellowish green. Fertile stamens 3, filaments glabrous. Staminodes 7, glabrous, 2 inserted individually between the stamens, the remaining 5 mounted on a raised, fleshy disc that is open at the lower end, with sterile antherodes, these caducous. Ovary glabrous, or with some hairs on basal part. Stigma capitate, peltate. Fruit compressed, not woody, narrowly to broadly oblong, glabrous, many seeded, indehiscent or tardily dehiscent. Seeds (10-)15-36 per fruit; with rim-like funicle extending along the majority of the seed circumference (known in 5 species). 


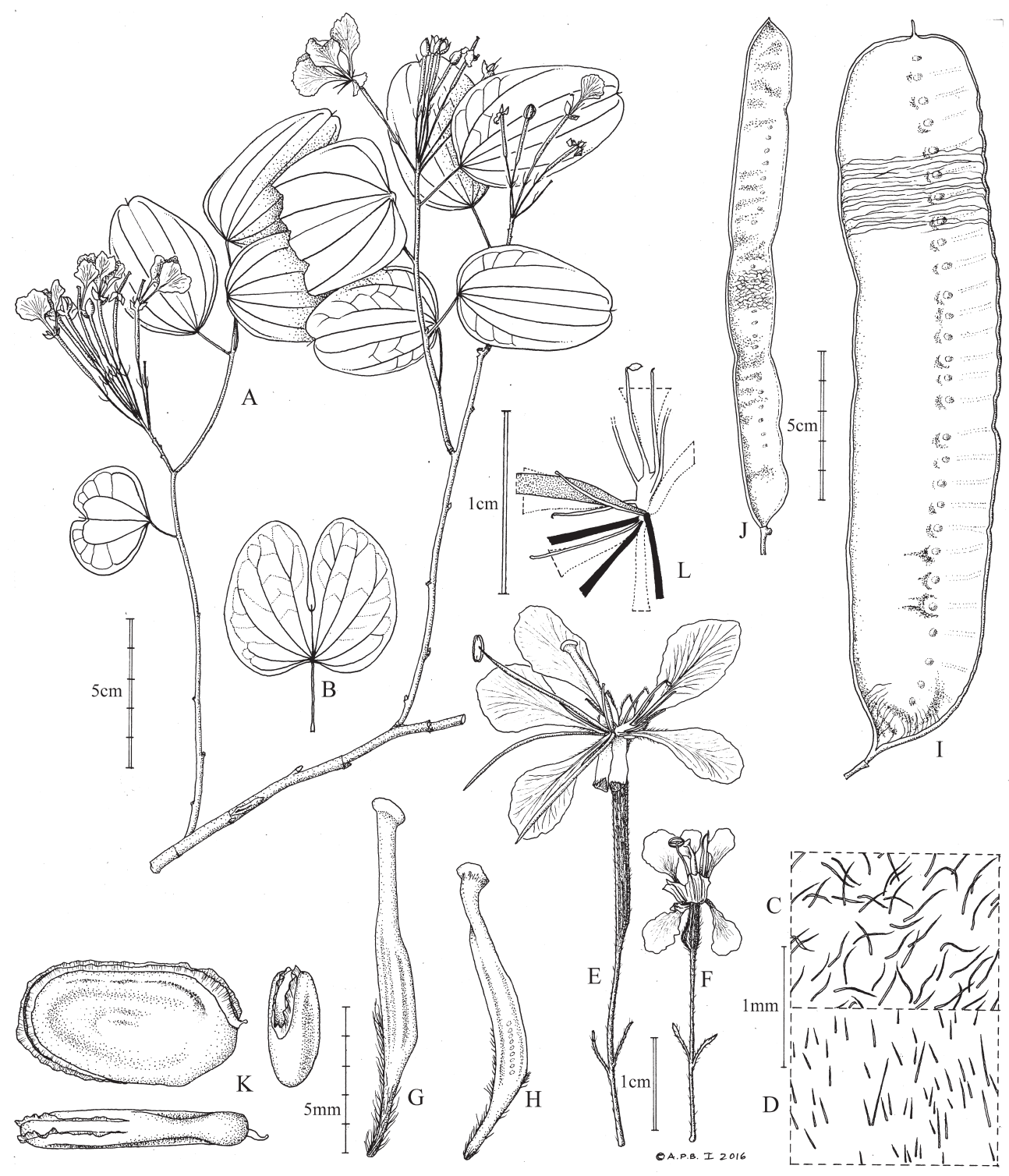

Fig. 4. Illustration of Cheniella R.Clark \& Mackinder gen. nov. A. Cheniella ovatifolia (T.C.Chen) R.Clark \& Mackinder comb. nov., habit (Harder et al. 4249). B. C. glauca (Wall. ex Benth.) R.Clark \& Mackinder comb. nov., leaf abaxial surface (Horsfield 165). C. C. quinnanensis subsp. villosa R.Clark \& Mackinder subsp. nov., leaf abaxial indumentum (Tu 924). D. C. quinnanensis (T.C.Chen) R.Clark \& Mackinder comb. nov. subsp. quinnanensis, leaf abaxial indumentum (Forrest 11844). E. C. tenuiflora (Watt ex C.B.Clarke) R.Clark \& Mackinder comb. nov., flower (Urquhart s.n.). F. C. touranensis (Gagnep.) R.Clark \& Mackinder comb. nov., flower (Henry 10175). G. C. touranensis, ovary (Harder \& al. 2387). H. C. quinnanensis, ovary (Bar 2676). I. C. tenuiflora, fruit (Winit 1455). J. C. corymbosa (Roxb.) R.Clark \& Mackinder comb. nov., fruit (Cult. Calcutta B.G. s.n.). K. C. corymbosa, seed, three views (Cult. Calcutta B.G. s.n.). L. C. tenuiflora, schematised flower, showing staminodes (2 broken), bases of 3 stamen filaments (solid black), base of ovary (dotted), bases of petals (dotted outlines), sepals and hairs omitted. Drawn by Andrew Brown. 
Key to the species of Cheniella gen. nov. (see comparison of key characters in Table 1)

1. Leaf entire to emarginate............................................................ 2

- Leaf bilobed to at least $1 / 10$ of length ....................................................... 4

2. Leaf on lower surface moderately to densely dark ferruginous tomentose, sparsely white tomentose on upper surface (or with few hairs at the base); petioles $0.5-1.4 \mathrm{~cm}$; inflorescence a raceme, densely ferruginous tomentose, the axis 5-13 cm; pedicels 1.3-1.6 cm; hypanthium (2-) $23-25 \mathrm{~mm}$

3. C. clemensiorum (Merr.) R.Clark \& Mackinder comb. nov.

- Leaf on lower surface sparsely to moderately pale ferruginous appressed hairy, tomentose, villose, or pilose on veins, upper surface glabrous (or with sparse hairs on the midvein only); petioles $0.8-2.8 \mathrm{~mm}$; inflorescence a raceme or corymb, the axis usually shorter than $10 \mathrm{~cm}$, sparsely to moderately pale tomentose; pedicels $1.5-3.3 \mathrm{~mm}$; hypanthium $7-20 \mathrm{~mm}$

\section{.} (1)

\section{,}


Table 1. Comparison of key characters of species and subspecies of Cheniella (significant characters distinguishing C. glauca and C. tenuiflora are highlighted in bold).

\begin{tabular}{|c|c|c|c|c|c|c|}
\hline & $\begin{array}{l}\text { C. clemensiorum } \\
\text { comb. nov. }\end{array}$ & $\begin{array}{l}\text { C. corymbosa } \\
\text { comb. nov. }\end{array}$ & $\begin{array}{l}\text { C. damiaosiao- } \\
\text { shanensis } \\
\text { comb. nov. }\end{array}$ & $\begin{array}{c}\text { C. didyma } \\
\text { comb. nov. }\end{array}$ & $\begin{array}{c}\text { C. glauca } \\
\text { comb. nov. }\end{array}$ & $\begin{array}{c}\text { C. lakhonensis } \\
\text { comb. nov. }\end{array}$ \\
\hline leaf apex & $\begin{array}{l}\text { rounded or } \\
\text { emarginate to } \\
1 \mathrm{~mm}\end{array}$ & bilobed to $2 / 3-7 / 8$ & bilobed to $1 / 4-1 / 3$ & $\begin{array}{l}\text { bilobed to } \\
\text { base, joined by } \\
1-2 \mathrm{~mm}\end{array}$ & $\begin{array}{c}\text { bilobed to }(1 / 3) \\
>1 / 2\end{array}$ & bilobed to $1 / 3-2 / 3$ \\
\hline leaf size $\mathrm{cm}$ & $5-7 \times 4-7$ & $2-4 \times 2-5$ & $2-5 \times 2.5-6$ & $1.2-2.5 \times 1.8-3.2$ & $3.1-8 \times 3.6-6.7$ & $2.4-5 \times 3.7-6.3$ \\
\hline $\begin{array}{l}\text { mucro length } \\
\text { mm }\end{array}$ & 2 & 2 & $0.5-1$ & $<1$ & $1-2$ & 2 \\
\hline $\begin{array}{l}\text { no. secondary } \\
\text { veins }\end{array}$ & $6-8$ & $4-6$ & $6-8$ & 6 & $8-10$ & $8-10$ \\
\hline $\begin{array}{l}\text { leaf } \\
\text { indumentum } \\
\text { upper }\end{array}$ & $\begin{array}{c}\text { sparsely } \\
\text { tomentose, or } \\
\text { few hairs at base } \\
\text { only }\end{array}$ & glabrous & glabrous & glabrous & $\begin{array}{l}\text { glabrous } \\
\text { to sparsely } \\
\text { tomentose }\end{array}$ & $\begin{array}{c}\text { glabrous or } \\
\text { sparsely pilose }\end{array}$ \\
\hline $\begin{array}{l}\text { leaf } \\
\text { indumentum } \\
\text { lower }\end{array}$ & $\begin{array}{l}\text { moderately to } \\
\text { densely villose }\end{array}$ & $\begin{array}{c}\text { sparsely } \\
\text { appressed pilose }\end{array}$ & pilose at base & $\begin{array}{c}\text { sparsely } \\
\text { pubescent on } \\
\text { primary and } \\
\text { secondary veins; } \\
\text { or subglabrous } \\
\text { with hairs } \\
\text { between base of } \\
\text { veins }\end{array}$ & $\begin{array}{c}\text { sparsely to } \\
\text { moderately } \\
\text { appressed hirsute }\end{array}$ & $\begin{array}{c}\text { sparsely to } \\
\text { moderately pilose }\end{array}$ \\
\hline $\begin{array}{l}\text { petiole length } \\
\mathrm{cm}\end{array}$ & $0.5-1.4$ & $0.8-2$ & $1.5-2$ & $1-2$ & $1.1-4.2$ & $0.8-2.8$ \\
\hline $\begin{array}{l}\text { inflorescence } \\
\text { type; axis length } \\
\text { cm }\end{array}$ & $\begin{array}{c}\text { raceme, } \\
\text { subcorymbose at } \\
\text { apex; 5-13 }\end{array}$ & corymb; $1.5-6$ & $\begin{array}{c}\text { raceme, lax and } \\
\text { few-flowered; } \\
6-8.5\end{array}$ & $\begin{array}{l}\text { raceme, } \\
\text { corymbose at } \\
\text { apex; ca } 6\end{array}$ & corymb; $2.5-4.5$ & raceme; 3-9 \\
\hline bract length mm & $5-11$ & $3-5$ & $4-7$ & $1.5-4$ & $5-6$ & $3-4(-6)$ \\
\hline $\begin{array}{l}\text { bracteole length } \\
\mathrm{mm}\end{array}$ & $5-7$ & $3-5$ & $2-4$ & $1.5-4$ & $4-5$ & $4-5$ \\
\hline $\begin{array}{l}\text { pedicel length } \\
\mathrm{cm}\end{array}$ & $1.3-1.6$ & $1-2.6$ & $1.8-2$ & $1-3.0$ & 1-1.6 & $0.7-1.7$ \\
\hline $\begin{array}{l}\text { hypanthium } \\
\text { length cm }\end{array}$ & $(2.0-) 2.3-2.5$ & $1.8-2.5$ & $0.6-0.9$ & {$[0.8-1.2]$} & $1.0-1.2[-1.5]$ & $2.0-2.8$ \\
\hline $\begin{array}{l}\text { hypanthium } \\
\text { indumentum }\end{array}$ & $\begin{array}{l}\text { moderately to } \\
\text { densely hirsute }\end{array}$ & $\begin{array}{c}\text { sparsely to } \\
\text { moderately } \\
\text { hirsute or pilose }\end{array}$ & $\begin{array}{l}\text { sparsely } \\
\text { appressed hirsute } \\
\text { or pilose }\end{array}$ & sparsely pilose & $\begin{array}{l}\text { glabrous, or } \\
\text { with few hairs } \\
\text { at base }\end{array}$ & $\begin{array}{c}\text { sparsely to } \\
\text { moderately pilose }\end{array}$ \\
\hline $\begin{array}{l}\text { hypanthium } \\
\text { inflated at base }\end{array}$ & no & no & no & no & no & no \\
\hline petal size mm & $8-10 \times 4-8$ & $8-13 \times 4-9$ & $5-8 \times 1.5-3$ & ca $9 \times 6$ & $8-10 \times 5-7$ & $8-14 \times 4-9$ \\
\hline $\begin{array}{l}\text { petal claw } \\
\text { length mm }\end{array}$ & ca 1 & $1-2$ & $1-4$ & unknown & $2-3$ & $1-3$ \\
\hline $\begin{array}{l}\text { stamen length } \\
\text { mm }\end{array}$ & $13-15$ & $8-10$ & $9-10$ & 10 & 8-10 & $8-10$ \\
\hline $\begin{array}{l}\text { staminodal disc } \\
\mathrm{I} \times \mathrm{W}\end{array}$ & $1 \times 2$ & $0.5 \times 2$ & $2-3 \times 1-2$ & unknown & $1 \times 2$ & $0.5 \times 1$ \\
\hline $\begin{array}{l}\text { staminode } \\
\text { length } \mathrm{mm}\end{array}$ & ca 4-6 & ca $2-4$ & $1-2$ & unknown & 2-3 & $2-3$ \\
\hline $\begin{array}{l}\text { ovary } \\
\text { indumentum }\end{array}$ & glabrous & glabrous & glabrous & glabrous & glabrous & glabrous \\
\hline fruit length $\mathrm{cm}$ & $16-23$ & $10-25$ & {$[12-15] \mathrm{cm}$} & ca 10 & $17.5-20.5$ & $10-12.5$ \\
\hline fruit width $\mathrm{cm}$ & $4-5$ & $1.5-2.5$ & {$[3-3.5]$} & ca 2.5 & $3.9-5.5$ & $1.7-2.3$ \\
\hline no. seeds & $20-36$ & $21-23$ & [15-18] & ca 20 & 17-19 & $15-21$ \\
\hline
\end{tabular}


Table 1 (continued).

\begin{tabular}{|c|c|c|c|c|c|c|}
\hline & $\begin{array}{l}\text { C. ovatifolia } \\
\text { comb. nov. }\end{array}$ & $\begin{array}{l}\text { C. quinnanensis } \\
\text { subsp. } \\
\text { gandhiana } \\
\text { comb. nov. }\end{array}$ & $\begin{array}{c}\text { C. quinnanensis } \\
\text { comb. nov. } \\
\text { subsp. } \\
\text { quinnanensis }\end{array}$ & $\begin{array}{l}\text { C. quinnanensis } \\
\text { subsp. villosa } \\
\text { subsp. nov. }\end{array}$ & $\begin{array}{l}\text { C. tenuiflora } \\
\text { comb. nov. }\end{array}$ & $\begin{array}{l}\text { C. touranensis } \\
\text { comb. nov. }\end{array}$ \\
\hline leaf apex & $\begin{array}{l}\text { rounded to } \\
\text { emarginate }\end{array}$ & $\begin{array}{l}\text { bilobed to ca } \\
10 \mathrm{~mm} \text {, or } \\
\text { attenuate }\end{array}$ & $\begin{array}{l}\text { emarginate to } \\
3 \mathrm{~mm}\end{array}$ & $\begin{array}{l}\text { emarginate to } \\
4 \mathrm{~mm}\end{array}$ & $\begin{array}{l}\text { bilobed to } 1 / 10 \\
\text { (or less)- } 1 / 2\end{array}$ & bilobed to $1 / 6-1 / 2$ \\
\hline leaf size $\mathrm{cm}$ & $3-7 \times 2-4.8$ & $4.7-10 \times 3-9$ & $5-10.5 \times 3.5-9$ & $5.2-7.7 \times 4.1-6.4$ & $4.2-9 \times 4.7-10.7$ & $2.5-6 \times 3-6.5$ \\
\hline $\begin{array}{l}\text { mucro length } \\
\text { mm }\end{array}$ & $1-2$ & unknown & $1-2$ & $1-2$ & $(1.5-) 2-4$ & 2 \\
\hline $\begin{array}{l}\text { no. secondary } \\
\text { veins }\end{array}$ & 6 & $6-8$ & $8(-10)$ & 10 & $6-10$ & $6-8$ \\
\hline $\begin{array}{l}\text { leaf } \\
\text { indumentum } \\
\text { upper }\end{array}$ & glabrous & glabrous & glabrous & $\begin{array}{l}\text { few hairs on } \\
\text { midvein }\end{array}$ & $\begin{array}{l}\text { glabrous } \\
\text { to sparsely } \\
\text { tomentose }\end{array}$ & glabrous \\
\hline $\begin{array}{l}\text { leaf } \\
\text { indumentum } \\
\text { lower }\end{array}$ & $\begin{array}{l}\text { pubescent on } \\
\text { veins and near } \\
\text { base }\end{array}$ & $\begin{array}{l}\text { pubescent, } \\
\text { particularly } \\
\text { on the veins, } \\
\text { glabrescent }\end{array}$ & $\begin{array}{c}\text { sparsely to } \\
\text { moderately } \\
\text { pilose, long and } \\
\text { short hairs }\end{array}$ & $\begin{array}{c}\text { sparsely to } \\
\text { moderately } \\
\text { villose, hairs } \\
\text { uniform, denser } \\
\text { on secondary } \\
\text { veins }\end{array}$ & $\begin{array}{c}\text { sparsely } \\
\text { appressed } \\
\text { hirsute, pilose, or } \\
\text { villose }\end{array}$ & $\begin{array}{c}\text { sparsely pilose, } \\
\text { or few hairs at } \\
\text { base only }\end{array}$ \\
\hline $\begin{array}{l}\text { petiole length } \\
\mathrm{cm}\end{array}$ & $0.8-2.5$ & $0.9-1.9$ & $(1-) 1.4-2.8$ & $1.2-1.5$ & $1.3-6$ & $1-3.2$ \\
\hline $\begin{array}{l}\text { inflorescence } \\
\text { type; axis length } \\
\text { cm }\end{array}$ & $\begin{array}{c}\text { subcorymbose; } \\
2.5-7\end{array}$ & $\begin{array}{l}\text { corymbose } \\
\text { raceme; length } \\
\text { unknown }\end{array}$ & $\begin{array}{l}\text { raceme, } \\
\text { corymbose at } \\
\text { apex; 4-8 }\end{array}$ & corymb, $2.5-5$ & $\begin{array}{c}\text { raceme, } \\
\text { corymbose at } \\
\text { apex; } 2.5-12\end{array}$ & $\begin{array}{l}\text { raceme, } \\
\text { corymbose at } \\
\text { apex; 4-8 }\end{array}$ \\
\hline $\begin{array}{l}\text { bract length } \\
\text { mm }\end{array}$ & $4-6$ & $8-9$ & $5-10$ & $5-6$ & $5-9$ & $6(-9)$ \\
\hline $\begin{array}{l}\text { bracteole length } \\
\mathrm{mm}\end{array}$ & $4-7$ & $8-9$ & $4-6$ & $5-6$ & $6-7$ & $4[-9]$ \\
\hline $\begin{array}{l}\text { pedicel length } \\
\mathrm{cm}\end{array}$ & $1.5-2.3$ & $3-3.6$ & $2.3-3.3$ & $1.8-2.2$ & $1.7-2.7$ & $2.1-2.5$ \\
\hline $\begin{array}{l}\text { hypanthium } \\
\text { length } \mathrm{cm}\end{array}$ & $1.5-2.0$ & ca 0.7 & $0.8-1.0$ & $1.5-2.0$ & $1.1-3.0$ & $0.4-0.6(-1.0)$ \\
\hline $\begin{array}{l}\text { hypanthium } \\
\text { indumentum }\end{array}$ & sparsely pilose & pubescent & $\begin{array}{c}\text { sparsely to } \\
\text { moderately } \\
\text { hirsute or pilose }\end{array}$ & $\begin{array}{l}\text { moderately to } \\
\text { densely villose }\end{array}$ & $\begin{array}{c}\text { sparsely to } \\
\text { moderately } \\
\text { (occasionally } \\
\text { densely) pilose }\end{array}$ & $\begin{array}{l}\text { sparsely to } \\
\text { moderately } \\
\text { pilose }\end{array}$ \\
\hline $\begin{array}{l}\text { hypanthium } \\
\text { inflated at base }\end{array}$ & very slightly & yes (slightly) & yes (slightly) & yes & no & yes (distinctly) \\
\hline petal size $\mathbf{m m}$ & $11-22 \times 6-15$ & $10-11 \times 4-5$ & $7-13 \times 4-10$ & $8-12 \times 5-10$ & $10-19 \times 5-14$ & $6-10 \times 2-4$ \\
\hline $\begin{array}{l}\text { petal claw } \\
\text { length } \mathbf{~ m m}\end{array}$ & $4-8$ & ca 3 & $2-4$ & $1-3$ & $2-5$ & $2-4$ \\
\hline $\begin{array}{l}\text { stamen length } \\
\text { mm }\end{array}$ & $7-12$ & $7-9$ & $9-13$ & $12-13$ & $12-17$ & $6-10$ \\
\hline $\begin{array}{l}\text { staminodal disc } \\
I \times w\end{array}$ & $1-2 \times 2$ & unknown & $2-3 \times 1-2$ & $1 \times 2$ & $1-2 \times 2$ & $1-2 \times 1$ \\
\hline $\begin{array}{l}\text { staminode } \\
\text { length } \mathrm{mm}\end{array}$ & $3-5$ & ca 2.5 & $2-3$ & ca 3 & $5-7$ & $1-2$ \\
\hline $\begin{array}{l}\text { ovary } \\
\text { indumentum }\end{array}$ & glabrous & $\begin{array}{c}\text { sparsely } \\
\text { pubescent on } \\
\text { sutures at base }\end{array}$ & $\begin{array}{l}\text { few hairs on } \\
\text { basal part of } \\
\text { lower suture }\end{array}$ & $\begin{array}{l}\text { few hairs on } \\
\text { basal } 1 / 2-3 / 4 \text { of } \\
\text { lower suture }\end{array}$ & $\begin{array}{c}\text { glabrous, or with } \\
\text { few hairs on stipe } \\
\text { or basal part of } \\
\text { suture }\end{array}$ & $\begin{array}{c}\text { stipe and basal } \\
\text { half of ovary } \\
\text { hairy }\end{array}$ \\
\hline fruit length cm & $20-25$ & unknown & $23-28$ & unknown & $16.5-27$ & {$[12-] 17-18$} \\
\hline fruit width $\mathrm{cm}$ & $4-4.5$ & unknown & $4-4.5(-5.5)$ & unknown & $3.3-5.6$ & {$[3-] 4.5$} \\
\hline no. seeds & numerous & unknown & ca 23 & unknown & {$[10-] 17-31$} & $15-26$ \\
\hline
\end{tabular}


1. Cheniella quinnanensis (T.C.Chen) R.Clark \& Mackinder comb. nov. urn:Isid:ipni.org:names:77165905-1

Basionym: Bauhinia quinnanensis T.C.Chen, Guihaia 8 (1): 48 (Chen 1988). - Type: China, Guizhou, Anlong, Shipan, 26 May 1960, Guizhou Expedit. 3053 (holo-: SCBI n.v.). - Additional material: China, Cehang, Guizhou Expedit. 2332, 2416 (para-: IBSC). China, Xingren, Dashui, 1,300 m a.s.1., 26 Jul. 1960, Guizhou Expedit. 8755 (para-: IBSC).

\section{Description}

Leaf entire, ovate, apex emarginate to ca $10 \mathrm{~mm}$, with mucro $1-2 \mathrm{~mm}$, or occasionally attenuate; $4.7-$ $10.5 \times(3.0-) 3.5-9.0 \mathrm{~cm}$; upper surface drying dark brown to blackish, glabrous, or with sparse hairs on the midvein, lower surface sparsely to moderately ferruginous semi-appressed pilose with short and long hairs, slightly denser at base, or sparsely to moderately villose, the hairs denser on the veins, particularly at the base; secondary veins $6-10$. Petiole [0.9- $] 1.4-2.8 \mathrm{~cm}$. Stipule $2-5 \mathrm{~mm}$. Inflorescence a corymb, or raceme becoming corymbose at the apex; axis $2.5-8.0 \mathrm{~cm}$. Pedicel $1.8-3.6 \mathrm{~cm}$. Bracts $5-10 \mathrm{~mm}$. Bracteoles 4-9 mm. Hypanthium (slightly) inflated at base, $0.7-2.0 \mathrm{~mm}$, sparsely to densely ferruginous pubescent, hirsute, pilose or villous. Petals white, ca 7-13 $\times 4-10 \mathrm{~mm}$, sparsely pilose on outer surface, hairs mostly close to midvein, claw 1-4 mm. Fertile stamens 3, each 7-13 mm. Staminodes 7, of which 2 are inserted individually between the stamens, 5 mounted on a raised fleshy disc that is open on the side where the stamens are inserted, disc 1-3 $\times 1-2 \mathrm{~mm}$, staminodes 2-3 mm. Ovary with a few hairs on stipe or on basal part of lower suture (or both), occasionally glabrous. Fruit broadly oblong, 23[-28] $\times$ $4.0-4.5(-5.5) \mathrm{cm}$. Seeds ca 23 per fruit, funicle encircling most of circumference of seed.

\section{Distribution}

China (Guangdong, Guangxi, Guizhou, Yunnan), India (Arunachal Pradesh, Assam), Myanmar.

\section{Ecology}

Open areas, scrub, roadside, forest; elevation 800-2,100 m a.s.1.

\section{Preliminary conservation assessment}

Extent of Occurrence 322,120.231 $\mathrm{km}^{2}=$ Least Concern (LC). Incorporating all subspecies, this species is distributed over a fairly wide area from southern China into northern Myanmar and India, and seems to be able to occupy a variety of habitats. It is therefore here considered to be Least Concern, although it should be noted that it is quite poorly represented in herbaria (ca 18 specimens known to the present authors), which could indicate that it is relatively uncommon within its range.

\section{Key to the subspecies of $C$. quinnanensis comb. nov.}

1. Leaf dimorphic, the apex emarginate, or attenuate, both forms occurring on a single branch

1.3. C. quinnanensis subsp. gandhiana (Gogoi \& Bandyop.) R.Clark \& Mackinder comb. et stat. nov.

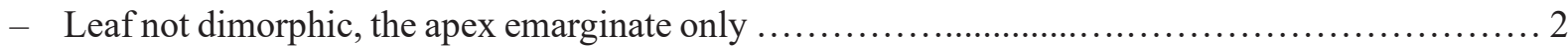

2. Hairs on lower leaf surface straight, mixed long and short, not or only slightly denser on secondary veins than blade; pedicel $23-33 \mathrm{~mm}$; hypanthium $0.8-1.0 \mathrm{~cm}$ long

1.1. C. quinnanensis (T.C.Chen) R.Clark \& Mackinder comb. nov. subsp. quinnanensis

- Hairs on lower leaf surface villose, length more or less uniform, much denser on secondary veins than blade; pedicel 18-22 $\mathrm{mm}$; hypanthium $1.5-2.0 \mathrm{~cm}$ long 1.2. C. quinnanensis subsp. villosa R.Clark \& Mackinder subsp. nov. 
1.1. Cheniella quinnanensis (T.C.Chen) R.Clark \& Mackinder comb. nov. subsp. quinnanensis

Phanera glauca Benth. subsp. tenuiflora (Watt ex C.B.Clarke) K.Larsen \& S.S.Larsen var. murlenensis Ram.Kumar, Bandyop. \& S.Sharma syn. nov., Phytotaxa 166 (2): 155 (Kumar et al. 2014). - Type: India, Mizoram, Murlen National Park, in the buffer region of the Park between Vapar to Ngur, ca 1,400 m a.s.1., 11 Apr. 2013, Ramesh Kumar \& party 128363 (holo-: CAL0000026092; iso-: ASSAM).

\section{Description}

Leaf 5.0-10.5 × 3.5-9.0 cm; upper surface drying dark brown, glabrous, lower surface sparsely to moderately ferruginous semi-appressed pilose with short and long hairs, slightly denser at base; secondary veins $8(-10)$. Pedicel $2.3-3.3 \mathrm{~cm}$. Hypanthium slightly inflated at base, $0.8-1.0 \mathrm{~mm}$; sparsely to moderately ferruginous hirsute or pilose.

\section{Distribution}

China (Guangxi, Yunnan, Guizhou), India (Assam), Myanmar.

\section{Ecology}

Open areas, scrub, roadside, forest; elevation 1,150-2,100 m a.s.1.

\section{Taxonomic and geographical notes}

Phanera glauca subsp. tenuiflora var. murlenensis is here synonymised with C. quinnanensis comb. nov. on the grounds that the description does not differ significantly from that of $C$. quinnanensis comb. nov., and that the specimen's locality is in Mizoram state in northern India, which is adjacent to Assam state in which C. quinnanensis comb. nov. subsp. quinnanensis is known to occur.

1.2. Cheniella quinnanensis subsp. villosa R.Clark \& Mackinder subsp. nov. urn:lsid:ipni.org:names:77165906-1

\section{Diagnosis}

Leaf 5.2-7.7 × 4.1-6.4 cm; upper surface drying glossy and blackish, glabrous apart from sparse hairs on the midvein, lower surface sparsely to moderately villose, the hairs denser on the veins, particularly at the base; secondary veins 10 . Inflorescence a corymb, axis $2.5-5 \mathrm{~cm}$. Pedicel $1.8-2.2 \mathrm{~cm}$. Hypanthium inflated at base, $1.5-2.0 \mathrm{~mm}$, moderately to densely ferruginous villose.

\section{Type}

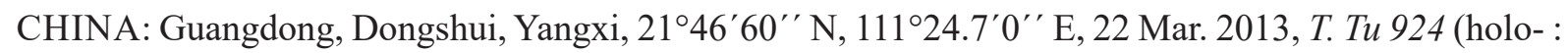
K000980298!; iso-: IBSC n.v.).

\section{Distribution}

Known only from the type specimen, collected in Guangdong Province. This province is adjacent to Guangxi, where C. quinnanensis comb. nov. subsp. quinnanensis occurs.

\section{Ecology}

Open area, near creek.

\section{Field notes}

Petals white, nectar guide yellow, turning red. 


\subsection{Cheniella quinnanensis subsp. gandhiana (Gogoi \& Bandyop.) \\ R.Clark \& Mackinder comb. et stat. nov. urn:lsid:ipni.org:names:77165907-1}

Basionym: Phanera glauca Benth. subsp. tenuiflora (Watt ex C.B.Clarke) K.Larsen \& S.S.Larsen var. gandhiana Gogoi \& Bandyop, Journal of the Botanical Research Institute of Texas 8 (1): 71 (Gogoi \& Bandyopadhyay 2014). - Type: India, Arunachal Pradesh, Anjaw District, in between Changwanti and Walong, 800 m a.s.l., 20 May 2011, R. Gogoi 24374 (holo-: CAL0000025085; iso-: ARUN, ASSAM).

\section{Description}

Leaf ovate or ovate-orbicular, $4.7-10 \times 3.0-9.0 \mathrm{~cm}$, retuse to attenuate, or bilobed to ca $10 \mathrm{~mm}$; glabrous above, pubescent beneath, particularly on the veins, later glabrescent except on the veins; secondary veins $6-8$.

\section{Distribution}

Endemic to India (Arunachal Pradesh).

\section{Ecology}

Tropical evergreen forest; elevation ca $800 \mathrm{~m}$ a.s.1.

\section{Taxonomic notes}

This taxon was described as Phanera glauca subsp. tenuiflora var. gandhiana; however, we consider the variety to be more closely aligned with $C$. quinnanensis comb. nov. than with $C$. glauca comb. nov. The leaves are variable, some are similar in size and shape to C. quinnanensis comb. nov., and the ovary bears hairs at the base as in C. quinnanensis comb. nov., whereas the ovary of C. glauca comb. nov. is completely glabrous. Moreover, the dimensions of the hypanthium, pedicel, and stamens are more congruent with those of $C$. quinnanensis comb. nov. than with the taxon recognised by those authors as Phanera glauca subsp. tenuiflora. The type collection of C. quinnanensis subsp. gandhiana comb. et stat. nov. is from Arunachal Pradesh state in northern India, which is adjacent to Assam state, in which C. quinnanensis comb. nov. subsp. quinnanensis is known to occur.

A noteable and unusual feature of this taxon is the leaf form, which is dimorphic. Leaves are ovate and emarginate, with the base rounded to subcordate, or ovate with the apex attenuate to several millimetres, and the base sub-hastate. Both leaf forms can occur on the same branch of an individual. The latter leaf form is very unusual (possibly unique) within Bauhinia s. lat., raising the possibility that this plant is an anomalous form of $C$. quinnanensis comb. nov.; however, in the absence of opportunity to study the material directly, or further evidence to support this hypothesis, we here maintain the distinct status of the taxon, reclassifying it as a subspecies of $C$. quinnanensis comb. nov.

2. Cheniella ovatifolia (T.C.Chen) R.Clark \& Mackinder comb. nov. urn:lsid:ipni.org:names:77165908-1

Basionym: Bauhinia ovatifolia T.C.Chen, Guihaia 8 (1): 50 (Chen 1988). - Type: China, Guangxi, Tianyang, $700 \mathrm{~m}$ a.s.1., 28 Dec. 1955, Expedit. 404 (holo-: SCBI n.v.; iso-: IBG. n.v.).

\section{Description}

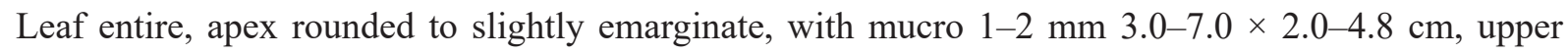
surface glabrous, lower surface pubescent on veins and near base; secondary veins 6 . Petiole $0.8-2.5 \mathrm{~cm}$. 
Stipules caducous, not seen. Inflorescence subcorymbose, axis $2.5-7.0 \mathrm{~cm}$. Pedicel $1.5-2.3 \mathrm{~cm}$. Bracts 4-6 mm. Bracteoles 4-7 mm. Hypanthium $1.5-2.0 \mathrm{~cm}$, sparsely ferruginous pilose, very slightly inflated at base. Petals white, 11-22 $\times 6-15 \mathrm{~mm}$, sparsely to moderately golden appressed pilose on outer surface, inner surface glabrous, claw prominent, 4-8 mm. Fertile stamens 3, each 7-12 mm. Staminodes 7, of which 2 are inserted individually between the stamens, 5 mounted on a raised fleshy disc that is open on the side where the stamens are inserted, disc 1-2 $\times 2 \mathrm{~mm}$, staminodes 3-5 mm. Ovary glabrous. Fruit $20-25 \times 4.0-4.5 \mathrm{~cm}$, glabrous. Seeds [numerous].

\section{Distribution}

China (Guangxi, Yunnan), Vietnam. The following specimen examined for this study represents a new species record for VIETNAM: Cao Bang, Tra Linh District, Quoc Toan Municipality, Thang Heng (Lakes District), $22^{\circ} 45^{\prime} 25^{\prime \prime} \mathrm{N}, 106^{\circ} 17^{\prime} 58^{\prime \prime}$ E, 666 m a.s.1., 6 Apr. 1998, D.K. Harder, P.K. Loc \& T.T. Tai 4249 (K000980301!).

\section{Ecology}

Open habitats on hill slopes; margin of disturbed forest on limestone; elevation 700-830 m a.s.1.

\section{Field notes}

Lianescent shrub to $4 \mathrm{~m}$; calyx green, striped pink and white; petals white; staminodes bright red at base to white at tip; filaments white; anthers red; sweetly fragrant.

\section{Preliminary conservation assessment}

Few herbarium specimens exist of this species, and it is not possible here to generate an EOO value due to limited access to locality data. The species is reported from Guangxi and Yunnan provinces (Dezhao et al. 2010), and from Vietnam; however, these two provinces are adjacent, and the Vietnamese specimen is from very near the border with Guangxi, so its total range may be small. The paucity of herbarium material available suggests that the species is uncommon. However, it must also be noted that the species occurs on limestone above $700 \mathrm{~m}$ a.s.l., which may constitute terrain that is difficult to access for collecting, and that this may account for the low number of specimens present in herbaria. We here consider the species to be Data Deficient (DD).

\section{Taxonomic notes}

The authors have been unable to access the type material of the species for this study, nor any positively verified material. A total of seven specimens identified as Bauhinia ovatifolia are available via the Chinese Virtual Herbarium, but their identity is in question because they are, with one exception, fruiting specimens, and have measurements that do not agree with those given in the protologue of the species. The data from these specimens has therefore not been included in this account. During this study, a flowering specimen (Harder et al. 4249) was examined by the authors and, based upon the description in the protologue, we believe that it represents C. ovatifolia comb. nov. Measurements of the Harder collection are included in the description given here, and the specimen has been used to create part of the illustration for the genus (Fig. 4).

3. Cheniella clemensiorum (Merr.) R.Clark \& Mackinder comb. nov. urn:lsid:ipni.org:names:77165909-1

Basionym: Bauhinia clemensiorum Merr., Journal of the Arnold Arboretum 23: 171 (Merrill 1942). Type: Vietnam, Annam, general vicinity of Hue and of Tourane, 1927, J. \& M.S. Clemens 3621 (holo- : A00059774 image!; iso-: BM000958843!, C10011873 image!, G00359963 image!, K000760828!, 
K000760829!, P00798452 image!, U0003152 image!, US00001272 image!). - Additional material: S. loc., 3 Jul. (fl), 3 Aug. (fr), J. \& M.S. Clemens 3229 (para-: Location of specimen unknown).

\section{Description}

Leaf entire, apex rounded to slightly emarginate (to $1 \mathrm{~mm}$ ), with mucro to $2 \mathrm{~mm}$; $5.0-7.0 \times 4.0-7.0 \mathrm{~cm}$; upper surface sparsely whitish tomentose (spreading hairs) or with just a few hairs at base; lower surface moderately to densely ferruginous villose, secondary veins $6-8$. Petiole $0.5-1.4 \mathrm{~cm}$. Stipules $5 \times 1 \mathrm{~mm}$. Inflorescence a raceme, subcorymbose at apex, axis 5-13 cm. Pedicel $1.3-1.6 \mathrm{~cm}$; densely ferruginous; pedicels persistent. Bracts 5-11 mm. Bracteoles 5-7 mm, persistent. Hypanthium (2-)2.3-2.5 cm; moderately to densely ferruginous hirsute. Petals white, $8-10 \times 4-8 \mathrm{~mm}$, outer surface sparsely to densely hairy, claw ca $1 \mathrm{~mm}$. Fertile stamens 3, each 13-15 mm. Staminodes 7, of which 2 inserted are individually between the stamens, 5 mounted on a raised fleshy disc that is open on the side where the stamens are inserted, disc $1 \times 2 \mathrm{~mm}$, staminodes $4-6 \mathrm{~mm}$. Ovary glabrous. Fruit broadly oblong, flat, glabrous, $16-23 \times 4.0-5.0 \mathrm{~cm}$. Seeds ca $20-36$ per fruit.

\section{Distribution}

Endemic to Vietnam.

\section{Ecology}

Evergreen closed forest on yellow ferralitic soil, forest margin; elevation ca $100 \mathrm{~m}$ a.s.1.

\section{Field notes}

Flowers pink-red.

\section{Preliminary conservation assessment}

Extent of Occurrence $1,140.324 \mathrm{~km}^{2}=$ Endangered (EN). Few herbarium specimens of this species are known: only four have been seen by the present authors, three of which were collected in the early 1900s, and no specimen records appear in the Chinese Virtual Herbarium. The 1997 IUCN Red List of Threatened Plants (Walter \& Gillett 1997), a list of species that were classified as being Rare, Vulnerable, Endangered, or Extinct included Bauhinia clemensiorum in the category Rare. Based on this combined evidence, we consider that the species should be classified as Endangered (E).

\section{Cheniella touranensis (Gagnep.) R.Clark \& Mackinder comb. nov. urn:lsid:ipni.org:names:77165910-1}

Basionym: Bauhinia touranensis Gagnep., Notulae systematicae. Herbier du Muséum de Paris. Phanérogamie 2 (6): 181 (Gagnepain 1912). - Type: Vietnam, Tonkin, environs de Tu-Phap, Feb. 1881, B. Balansa 2133 (lecto-, here designated: P00798502 image!; isolecto-: P00798503 image!). Remaining syntypes: Vietnam, Annam, Tourane, Jan. 1837, Gaudichaud 260 (P00798501 image!). Vietnam, Tonkin, d'Alleizette 494 (location of specimen unknown). Vietnam, Tonkin, T-Phap, May 1887, B. Balansa 2134 (K000760800!). Vietnam, baie d'Along, B. Balansa 1286 (location of specimen unknown). Vietnam, environs de Ninh-binh, Bon 2513, 2607, 6193 (location of specimen unknown).

\section{Description}

Leaf bilobed to $1 / 6-1 / 2$, with mucro $2 \mathrm{~mm}, 2.5-6.0 \times 3.0-6.5 \mathrm{~cm}$; upper surface glabrous, lower surface with few long orange hairs at base and on basal portion of veins, or with sparse long hairs all over, denser tuft between base of veins; secondary veins 6-8. Petiole $1.0-3.2 \mathrm{~cm}$. Stipules 5-6 $61.5 \mathrm{~mm}$. 
Inflorescence a raceme, corymbose at the apex, axis $4-8 \mathrm{~cm}$. Pedicel $2.1-2.5 \mathrm{~cm}$. Bracts ca 6(-9) $\mathrm{mm}$. Bracteoles ca 4[-9] mm. Hypanthium 0.4-0.6(-1.0) $\mathrm{cm}$, distinctly inflated at base, sparsely to moderately ferruginous pilose. Petals lime green to greenish yellow, 6-10 $\times 2-4 \mathrm{~mm}$, outer surface sparsely appressed pilose, hairs mostly along central vein (sometimes moderately dense at base), hairs pale orange, claw 2-4 mm. Fertile stamens 3, each 6-10 $\mathrm{mm}$. Staminodes 7, of which 2 are inserted individually between the stamens, 5 mounted on a raised fleshy disc that is open on the side where the stamens are inserted, disc 1-2 $\times 1 \mathrm{~mm}$, staminodes 1-2 mm. Ovary partially hairy, with stipe moderately hairy, hairs extending along basal half of ovary, densest on lower suture, sometimes a few on style just below stigma. Fruit broadly oblong, truncate at apex, cuneate at base; [12-]17-18 $\times[3.0-] 4.5 \mathrm{~cm}$. Seeds $15-26$ per fruit.

\section{Distribution}

China (Guangxi, Guizhou, Yunnan), Laos, Myanmar, Vietnam.

\section{Ecology}

Primary lowland forest, primary broad-leaved evergreen closed wet forest on limestone, disturbed agricultural fields, gorge, over rocks; elevation to $1,100 \mathrm{~m}$ a.s.1.

\section{Preliminary conservation assessment}

Extent of Occurrence 262,775.118 $\mathrm{km}^{2}=$ Least Concern (LC). This species is fairly well represented in herbaria. It is distributed throughout northern Vietnam, extending into central Laos. Its primary habitat is forest, but it seems also able to inhabit open or disturbed areas. It is here considered to be of Least Concern (LC) due to its fairly broad range and apparent suitability to different habitat types.

\section{Taxonomic notes}

The lectotype was selected for being the most complete specimen of the syntypes housed at Paris, where the author of the basionym worked, and where the majority of his collections are housed.

5. Cheniella corymbosa (Roxb.) R.Clark \& Mackinder comb. nov. urn:Isid:ipni.org:names:77165911-1

Basionym: Bauhinia corymbosa Roxb., Flora Indica 2: 329 (Roxburgh 1832); Hortus bengalensis: 31 (Roxburgh 1814) nom. nud. - Type: India, cult. Jard. Bot. de Calcutta, 1832, from seed of specimen W. Kerr s.n., 1796, China, Wallich 5788 (lecto- [first-step], designated by Larsen et al. (1980): K [3 sheets: K001122122!, K000760722!, K000760726!]; lecto- [second-step], here designated: K001122122!; isolecto-: K000760722!, K000760726!)).

\section{Description}

Leaf deeply bilobed to $2 / 3-7 / 8$, with mucro ca $2 \mathrm{~mm}, 2.0-4.0 \times 2.0-5.0 \mathrm{~cm}$; upper surface glabrous, lower surface sparsely appressed ferruginous pilose; secondary veins $4-6$. Petiole $0.8-2.0 \mathrm{~cm}$. Stipules $2 \mathrm{~mm}$, lanceolate. Inflorescence a corymb, axis $1.5-6.0 \mathrm{~cm}$. Pedicel $1.0-2.6 \mathrm{~cm}$. Bracts $3-5 \mathrm{~mm}$. Bracteoles 3-5 mm. Hypanthium 1.8-2.5 cm, sparsely to moderately ferruginous hirsute or pilose. Petals white with pink stripes, or pink, 8-13 $\times 4-9 \mathrm{~mm}$, sparsely to moderately ferruginous sericeous on outer surface, claw ca 1-2 mm. Fertile stamens 3, each 8-10 mm. Staminodes 7, of which 2 are inserted individually between the stamens, 5 mounted on a raised fleshy disc that is open on the side where the stamens are inserted, disc $0.5 \times 2 \mathrm{~mm}$, staminodes $2-4 \mathrm{~mm}$. Ovary glabrous. Fruit narrowly oblong, straight or falcate, drying black, sometimes opening along ventral (lower) suture in herbarium material, $10-25 \times$ $1.5-2.5 \mathrm{~cm}$. Seeds $21-23$ per fruit, funicle encircling most of seed circumference. 


\section{Distribution}

China (Hainan, Hong Kong, Guangdong), Vietnam (Tonkin).

\section{Ecology}

Forested ravines; elevation to $350 \mathrm{~m}$ a.s.1.

\section{Preliminary conservation assessment}

Extent of Occurrence 120,019.364 $\mathrm{km}^{2}=$ Least Concern (LC). Cheniella corymbosa comb. nov. is known from southern China and northern Vietnam. Based on the EOO generated here, and the fact that it is moderately well represented in herbaria (more than 20 specimens have been seen for this study), the species is here considered to be of Least Concern (LC). Little is known about its ecology, but it is known to occur in forest at low altitudes, and may therefore be vulnerable to habitat destruction. Additionally, many of the specimens seen for this study are old (pre-1950), and the populations from which they were taken may no longer exist. A more detailed study of the conservation status of this species should be undertaken when further data becomes available.

\section{Taxonomic Notes}

The lectotype was selected from the three sheets of Wallich 5788 for being the most complete specimen, bearing leaves and both flowers and fruit, whilst K000760722 also bears these but with fewer flowers, and K000760726 bears only leaves and fruit.

6. Cheniella lakhonensis (Gagnep.) R.Clark \& Mackinder comb. nov. urn:1sid:ipni.org:names:77165912-1

Basionym: Bauhinia lakhonensis Gagnep., Notulae systematicae. Herbier du Muséum de Paris. Phanérogamie 2 (6): 173 (Gagnepain 1912). - Type: Laos, La-khon, Vien-chang, Bang-muc, Thorel 3068 (syn-: P00798533 image!). Laos, Phon-thane, Spire 181 (syn-: Location of specimen unknown).

\section{Description}

Leaf bilobed to $1 / 3$ to $2 / 3$, with mucro ca $2 \mathrm{~mm}, 2.4-5.0 \times 3.7-6.3 \mathrm{~cm}$, upper surface glabrous or sparsely ferruginous pilose, lower surface sparsely to moderately ferruginous pilose; secondary veins 8-10. Petiole $0.8-2.8 \mathrm{~cm}$. Stipules ca $2 \mathrm{~mm}$. Inflorescence a raceme, becoming corymbose at the apex, axis $3.0-9 \mathrm{~cm}$. Pedicel 0.7-1.7 cm. Bracts 3-4(-6) mm. Bracteoles 4-5 mm. Hypanthium 2.0-2.8 cm, sparsely to moderately ferruginous pilose. Petals white, claws pink, 8-14 $\times 4-9 \mathrm{~mm}$, sparsely pilose/ tomentose on outer surface, claw 1-3 mm. Fertile stamens 3, each ca 8-10 mm. Staminodes 7, of which 2 are narrow, inserted individually between the stamens, 5 mounted on a raised fleshy disc that is open on the side where the stamens are inserted, disc $0.5 \times 1 \mathrm{~mm}$, staminodes $2-3 \mathrm{~mm}$. Ovary glabrous. Fruit oblong, rounded at the apex, base cuneate, $10-12.5 \times 1.7-2.3$. Seeds ca 15-21 per fruit.

\section{Distribution}

Laos, Thailand, China (Yunnan). The following specimen represents the first record of this species for CHINA: Yunnan, Lincang, 20 m a.s.1., 9 Feb. 2017, Tu, Tieyao 4010 (fl.) (IBSC, K).

\section{Ecology}

Roadside, mixed deciduous forest; elevation to 1,200 m a.s.1.

\section{Field notes}

Staminodes and filaments pink, anthers yellow-brown, stigma pink. 


\section{Preliminary conservation assessment}

Extent of Occurrence $146,856.735 \mathrm{~km}^{2}=$ Least Concern (LC). The species is found primarily in an area of southern-central Laos, and northern Thailand, with a single record in western Vietnam (near the boder with Laos), and a single record from Yunnan province, China. Without this recently discovered Chinese specimen, the EOO of the species would be considerably smaller, at $44,776.691 \mathrm{~km}^{2}$, in the category of Near Threatened (NT). However, this specimen shows that the species is more widespread than previously realised, and it is therefore considered here to be Least Concern (LC). It should be noted, however, that the habitat of $C$. lakhonensis is lowland forest, which may render it vulnerable to loss of populations due to the prevalence of deforestation in lowland areas.

\section{Taxonomic notes}

Larsen et al. (1980) stated the type to be Thorel s.n. from Thailand (P). It is not possible here to lectotypify the name as the present authors have not seen both syntypes.

7. Cheniella didyma (H.Y.Chen) R.Clark \& Mackinder comb. nov. urn:Isid:ipni.org:names:77165913-1

Basionym: Bauhinia didyma H.Y.Chen., Journal of the Arnold Arboretum 19: 131 (Chen 1938). Type: China, Kwangtung, Yeungchun, 16 Nov. 1935, Wang 38777 (fl, fr) (syn-: IBK00068594!, IBSC00044775, IBSC0004477, OC, PE00323954 image!, PE00323955 image!).

\section{Description}

Leaf deeply bilobed to base, the two lobes joined only by $1-2 \mathrm{~mm}$, with mucro $<1 \mathrm{~mm}, 1.2-2.5 \times$ 1.8-3.2 cm, upper surface glabrous, lower surface sparsely pubescent on primary and secondary veins, or mostly glabrous with tufts of hairs between base of secondary veins; secondary veins 6 . Petiole 1.0-2.0 cm. Stipules caducous (not seen). Inflorescence a raceme, corymbose at apex, axis ca $6.0 \mathrm{~cm}$. Pedicel 1-3 cm. Bracts 1.5-4 mm. Bracteoles 1.5-4 mm. Hypanthium [0.8-1.2] cm, sparsely pilose. Petals white, ca $9 \times 6 \mathrm{~mm}$, outer surface hairy near base. Fertile stamens 3 , each ca $10 \mathrm{~mm}$. Staminodes 7, of which 2 are narrow, inserted individually between the stamens, 5 mounted on a raised fleshy disc that is open on the side where the stamens are inserted, disc size unknown, staminodes 1-2 mm. Ovary glabrous. Fruit broadly oblong, ca $10 \times 2.5 \mathrm{~cm}$. Seeds ca 20 per fruit.

\section{Distribution}

Endemic to China (Guangdong, Guangxi, Hainan).

\section{Ecology}

Open forest, thickets; elevation 100-500 m a.s.1.

\section{Preliminary conservation assessment}

This species occurs in Guangdong, Guangxi, and Hainan Provinces, and is thus relatively broadly distributed. It is not possible here to generate an EOO value for the species due to limited access to locality data. However, the fact that few herbarium specimens exist (10 known to the present authors) suggests that it is uncommon. If further information were available, it might be expected that a category of threat would be applicable, although the fact that the species occurs in three provinces implies that the category of threat would not be high. Given the lack of locality information available for C. didyma comb. nov., it is here determined to be Data Deficient (DD). 


\section{Taxonomic notes}

Lectotypification has not been carried out as the authors have not seen all of the syntypes, and of those seen, none are flower-bearing.

The number of staminodes reported here derives from the protologue of Bauhinia didyma. Due to lack of access to material, the present authors have not been able to verify this. However, given that the number of staminodes in all other species of Cheniella gen. nov. is seven, it is likely that the number was misreported in the protologue, and that this species also possesses seven. It is presumed that some of the staminodes are mounted onto a raised fleshy disc as with the other species in the genus, altough it has not been possible to observe this.

8. Cheniella damiaoshanensis (T.C.Chen) R.Clark \& Mackinder comb. nov. urn:lsid:ipni.org:names:77165914-1

Basionym: Bauhinia damiaoshanensis T.C.Chen, Guihaia 8 (1): 49 (Chen 1988). - Type: China, Guangxi, Damiaoshan, Jiuwendashan, Luodong, Jun. 1957, T.C. Chen 537 (holo-: IBSC0088136 image!; iso- : IBG, K000760719!, MO104001 image!). - Additional material: China, Guangxi, Damiaoshan, Jiuwendashan, Luodong, S.H. Chun 15695 (para-: HITBC image!, IBSC image!). Ibid., C.H. Lui 2679 (para-: HITBC image!, IBSC image!). China, Damiaoshan, Sanfeng, R.C. Ching 5909 (para- : Location of specimen unknown). China, Darangjiang, R.C. Ching 6280 (para-: IBSC image!). China, Lingyun, Yuhong, S.K. Lau 28556 (para-: IBSC image!).

\section{Description}

Leaf bilobed to $1 / 4-1 / 3$ of length, mucro $0.5-1 \mathrm{~mm}, 2.0-5.0 \times 2.5-6.0 \mathrm{~cm}$, upper surface glabrous; lower surface ferruginous pilose at base, otherwise glabrous; secondary veins $6-8$. Petiole $1.5-2.0 \mathrm{~cm}$. Stipules linear, ca $2 \mathrm{~mm}$. Inflorescence a raceme, relatively lax and few-flowered, axis $6.0-8.5 \mathrm{~cm}$. Pedicel $1.8-$ $2.0 \mathrm{~cm}$. Bracts 4-7 mm. Bracteoles 2-4 mm. Hypanthium 0.6-0.9 cm, sparsely to moderately appressed ferruginous hirsute or pilose. Petals white, 5-8 $\times 1.5-3 \mathrm{~mm}$, densely appressed orange sericeous on outer surface, claw 1-4 mm. Fertile stamens 3, each 9-10 mm. Staminodes 7, of which 2 are inserted individually between the stamens, 5 mounted on a prominent raised fleshy disc up to $3 \mathrm{~mm}$ in length that is open on the side where the stamens are inserted, disc 2-3 $\times 1-2 \mathrm{~mm}$, staminodes 1-2 $\mathrm{mm}$. Ovary glabrous. Fruit [12-15] $\times[3.0-3.5] \mathrm{cm}$. Seeds [15-18] per fruit.

\section{Distribution}

Endemic to China (Guangxi).

\section{Ecology}

Open forests; elevation to $1,850 \mathrm{~m}$ a.s.1.

\section{Preliminary conservation assessment}

This species is endemic to Guangxi Province. It is not possible to generate an EOO value for the species due to limited access to locality data. However, the facts that it is known to occur in only one province, and that few herbarium specimens exist (ca 10 known to the present authors) suggests that it is uncommon. If further information were available it is likely that a category of threat would be applicable to the species. Until such time, it is here listed as Data Deficient (DD). 


\section{Cheniella tenuiflora (Watt ex C.B.Clarke) R.Clark \& Mackinder comb. nov. urn:Isid:ipni.org:names:77165915-1}

Basionym: Bauhinia tenuiflora Watt ex C.B.Clarke, The journal of the Linnean Society. Botany 25: 18 (Clarke 1889). - Bauhinia glauca subsp. tenuiflora (Watt ex C.B.Clarke) K.Larsen \& S.S.Larsen, Natural history bulletin of the Siam Society 25 (1-2): 10 (Larsen \& Larsen 1973). - Type: India, Muneypore, Nongjaibang, 30 Nov. 1885, C.B. Clarke 42304E (lecto- (Bandyopadhyay \& Sharma 1992): K000980300!). - Remaining syntypes: India, West Muneypore, 1500-2500 ft., s. coll. 42304A (K000980296!). Ibid., s. coll. 42304B (CAL). Ibid., s. coll. 42304C (K000980297!). Ibid., s. coll. $42304 D$ (CAL). Ibid., s. coll. 42255, 42342 (location of specimen unknown).

\section{Description}

Leaf shallowly bilobed to $1 / 10$ (or less) to $1 / 2$, with mucro (1.5-) 2 to $4 \mathrm{~mm}, 4.2-9.0 \times 4.7-10.7 \mathrm{~cm}$; upper surface glabrous or with few hairs at base, or sparsely tomentose, lower surface sparsely appressed ferruginous hirsute, pilose, or villose; secondary veins 6-10. Petiole 1.3-6.0 cm. Stipules 4-6 mm. Inflorescence a raceme, corymbose at apex, axis $2.5-12 \mathrm{~cm}$. Pedicel $1.7-2.7 \mathrm{~cm}$. Bracts $5-9 \mathrm{~mm}$. Bracteoles 6-7 mm. Hypanthium 1.1-3.0 cm, sparsely to moderately, occasionally densely, ferruginous pilose. Petals white to yellowish or pinkish, 10-19 $\times 5-14 \mathrm{~mm}$, moderately to densely ferruginous hairy on basal portion, densest around midvein, sometimes extending (sparsely) across whole blade, claw 2-5 mm. Fertile stamens 3, each 12-17 mm. Staminodes 7, of which 2 are inserted individually between the stamens, 5 mounted on a raised fleshy disc that is open on the side where the stamens are inserted, disc 1-2 $\times 2 \mathrm{~mm}$, staminodes 5-7 mm. Ovary glabrous or with few hairs on stipe or basal part of lower suture (both can occur on a single plant). Fruit broadly oblong, apex truncate, base rounded, 16.5-27 $\times$ $3.3-5.6 \mathrm{~cm}$. Seeds (10-)17-31 per fruit, funicle encircling most of the seed circumference.

\section{Distribution}

Cambodia, China (Guangxi, Guizhou, Guangdong, Hong Kong, Hainan, Hunan, Hubei, Nanto, Shaanxi, Szechuan, Yunnan), India (Assam, Manipur), Laos, Myanmar, Thailand, Vietnam (Fig. 10).

\section{Ecology}

Tropical evergreen forest, roadsides and open areas, thicket, disturbed hillside, mixed forest; elevation to $1,400 \mathrm{~m}$ a.s.1.

\section{Preliminary conservation assessment}

Extent of Occurrence 3,592,509.952 $\mathrm{km}^{2}=$ Least Concern (LC). This is a widespread and apparently common species, found from central China to east India and the Gulf of Thailand, and is therefore considered here to be Least Concern (LC).

\section{Taxonomic notes}

Specimens C.B. Clarke 42304A, 42304B, $42304 C$ and $42304 D$ can be considered as remaining syntypes following the lectotypification by Bandyopadhyay \& Sharma (1992) of C.B. Clarke 42304E, because C.B. Clarke 42304 was cited in the protologue without the associated letters A, B, etc., and thus could refer to any of these specimens. Larsen et al. (1980) cited the type of Bauhinia glauca subsp. tenuiflora to be Watt 6915 (Birmanie [Burma], E00064065!, K000760782!), but this is erroneous as this specimen is not one of the syntypes listed in the protologue.

The decision to recognise this taxon at the species level, rather than as a subspecies of Bauhinia glauca as it was designated by Larsen et al. (1980), was based on morphological differences, and on geographical separation (see Discussion). The morphological differences, highlighted in Table 1, consist of the 
indumentum or lack thereof on the hypanthium, the much more deeply bilobed leaf of C. glauca comb. nov., and differences between the size ranges of the floral parts and fruit characters. The differences between the two taxa are at least as great as those separating other species in the genus, and were therefore deemed to be sufficient to merit the recognition of this taxon as a distinct species. Moreover, Larsen et al. (1980) did not explain their reasons for choosing to down-rank Bauhinia tenuiflora, as it was originally described, to a subspecies of Bauhinia glauca.

10. Cheniella glauca (Wall. ex Benth.) R.Clark \& Mackinder comb. nov. urn:Isid:ipni.org:names:77165916-1

Basionym: Phanera glauca Wall. ex Benth., Plantae junghuhnianae 2: 265 (Miquel 1852). - Type: Myanmar, Amherst, 1827, Wallich catalogue no. 5785 (lecto-, here designated: K001122119!; isolecto-: K000760783!).

\section{Description}

Leaf bilobed to $1 / 3$ to (usually) more than $1 / 2$, with mucro $1-2 \mathrm{~mm}, 3.1-8.0 \times 3.6-6.7 \mathrm{~cm}$, upper surface glabrous, or sparsely tomentose with short, whitish, spreading hairs, lower surface sparsely to moderately ferruginous appressed hirsute, with denser tufts at base between veins; secondary veins 8-10. Petiole $1.1-4.2 \mathrm{~cm}$. Stipules 4-6 $\times 0.5-0.75 \mathrm{~mm}$. Inflorescence a corymb, axis $2.5-4.5 \mathrm{~cm}$. Pedicel $1.0-1.6 \mathrm{~cm}$. Bracts 5-6 mm. Bracteoles 4-5 mm. Hypanthium 1.0-1.2 [-1.5] cm, glabrous or sometimes with few hairs at base. Petals white to light yellow, $8-10 \times 5-7 \mathrm{~mm}$, sparsely pale orange sericeous on outer surface, claw 2-3 mm. Fertile stamens 3, each 8-10 mm. Staminodes 7, of which 2 are inserted individually between the stamens, 5 mounted on a raised fleshy disc that is open on the side where the stamens are inserted, disc $1 \times 2 \mathrm{~mm}$, staminodes $2-3 \mathrm{~mm}$. Ovary glabrous. Fruit broadly oblong, truncate to rounded at apex, rounded at base, $17.5-20.5 \times 3.9-5.5 \mathrm{~cm}$. Seeds ca $17-19$ per fruit.

\section{Distribution}

Java, Myanmar, Peninsular Malaysia (Pahang, Perak), Sumatra, Thailand (Fig. 10). The species has been reported from India (Bandyopadhyay 2001), but that lone report is most probably erroneous.

\section{Ecology}

Open roadsides, by the sea, rain forest, in ravines and on slopes, evergreen forest, scrub; elevation to $1,700 \mathrm{~m}$ a.s.1.

\section{Preliminary conservation assessment}

Extent of Occurrence $920,396.728 \mathrm{~km}^{2}=$ Least Concern (LC). This species is distributed over a fairly broad range, in several countries, and is represented by numerous herbarium specimens. It is therefore considered to be Least Concern (LC).

\section{Taxonomic notes}

The lectotype was selected for being the more complete specimen of the two, with more numerous and better preserved leaves.

Bauhinia tenuiflora, formerly considered to be a subspecies of Bauhinia glauca, is here recognised as a distinct species, the reasons for which are outlined in the text for that taxon. 


\section{Discussion}

\section{Specialised morphology of Cheniella gen. nov. (1): Pollination syndrome}

As discussed in the description of the pollen of the Corymbosae earlier in this paper, the pollen type of Cheniella gen. nov. is indicative of pollination by butterflies, moths, birds or bats. Here we explore further evidence to explain the pollination syndrome of the genus.

The primary pollinators in the legume family are bees, the majority of these being the long-tongued Apidae and Megachilidae (Lewis et al. 2000). Butterfly, moth, bird and bat pollination are also known to occur within the family, including subfam. Cercidoideae, and within Bauhinia s. lat. (Hokche \& Ramírez 1990; Lewis et al. 2000).

With regard to pollination syndromes, caesalpinioid legume flowers are in most cases less specialised than mimosoid or papilionoid flowers. Within the Caesalpinioideae (excluding the mimosoid clade), the flowers are usually open, with exposed pollen and nectar available to both specialised and nonspecialised pollen vectors (Arroyo 1981). However, a notable specialisation of the flowers of Cheniella gen. nov. is the hypanthium, which is as long as, or longer than, the calyx lobes, and is in absolute terms longer than the hypanthium of most other species of Phanera s. lat. Selective effects of pollinators are known to generate differences in flower morphology (Lewis 1998; Pérez-Barrales et al. 2007), and in this case the elongated hypanthium of Cheniella gen. nov. is suggestive of a specialised relationship with particular species (or groups) of pollinator.

It is documented that nectar is produced and accumulated in the hypanthium of flowers in the arborescent Bauhinia species (Hokche \& Ramírez 1990), and the elongated hollow hypanthium of Cheniella gen. nov. is presumed to serve similarly as a nectar receptacle. Flowers that are specialised to attract bees or other insects with long tongues often have petals fused into a tube, and are typically "high reward" flowers, offering more nectar than open access flowers (O'Toole \& Raw 1991). In the case of Cheniella gen. nov., restricting access to nectar, a function served in other flowers by a tubular corolla, could be served by the enlongated hypanthium. The long hypanthium of Cheniella gen. nov. is therefore most probably functionally related to particular pollinators, which are most likely to be long-tongued bees, moths or butterflies.

Evidence to support the hypothesis of pollination of the Corymbosae by bees and butterflies was provided by Lau et al. (2009), who studied visitation rate to the flowers of Bauhinia corymbosa and Bauhinia glauca by invertebrates. They noted that the bright colour of the stamens and carpel in Bauhinia corymbosa, diurnal anthesis, low viscosity of the nectar, and the dense multi-flowered inflorescences are all typical of butterfly-pollinated flowers, and that the flower characteristics of Bauhinia glauca are consistent with bee and butterfly pollination. The sucrose dominated nectar of both Bauhinia corymbosa and Bauhinia glauca is consistent with pollination by long-tongued bees and butterflies. Lau et al. (2009) observed that C. corymbosa comb. nov. was visited primarily by one species of bee (Hymenoptera), and by three species of butterfly (Lepidoptera), whilst Bauhinia glauca was visited by two species of bee and four species of butterfly. Their findings in terms of frequency of pollinator visits were that the flowers of Bauhinia corymbosa and Bauhinia glauca received significantly fewer visitations from potential pollinators than did the flowers of Bauhinia championii. A possible reason for this is that the flowers of Bauhinia corymbosa and Bauhinia glauca produce less nectar than those of Bauhinia championii, or that the nectar they produce is less accessible to a range of pollinators. This is compatible with the hypothesis that a longer hypanthium represents a specialised relationship with particular long-tongued pollinators. 


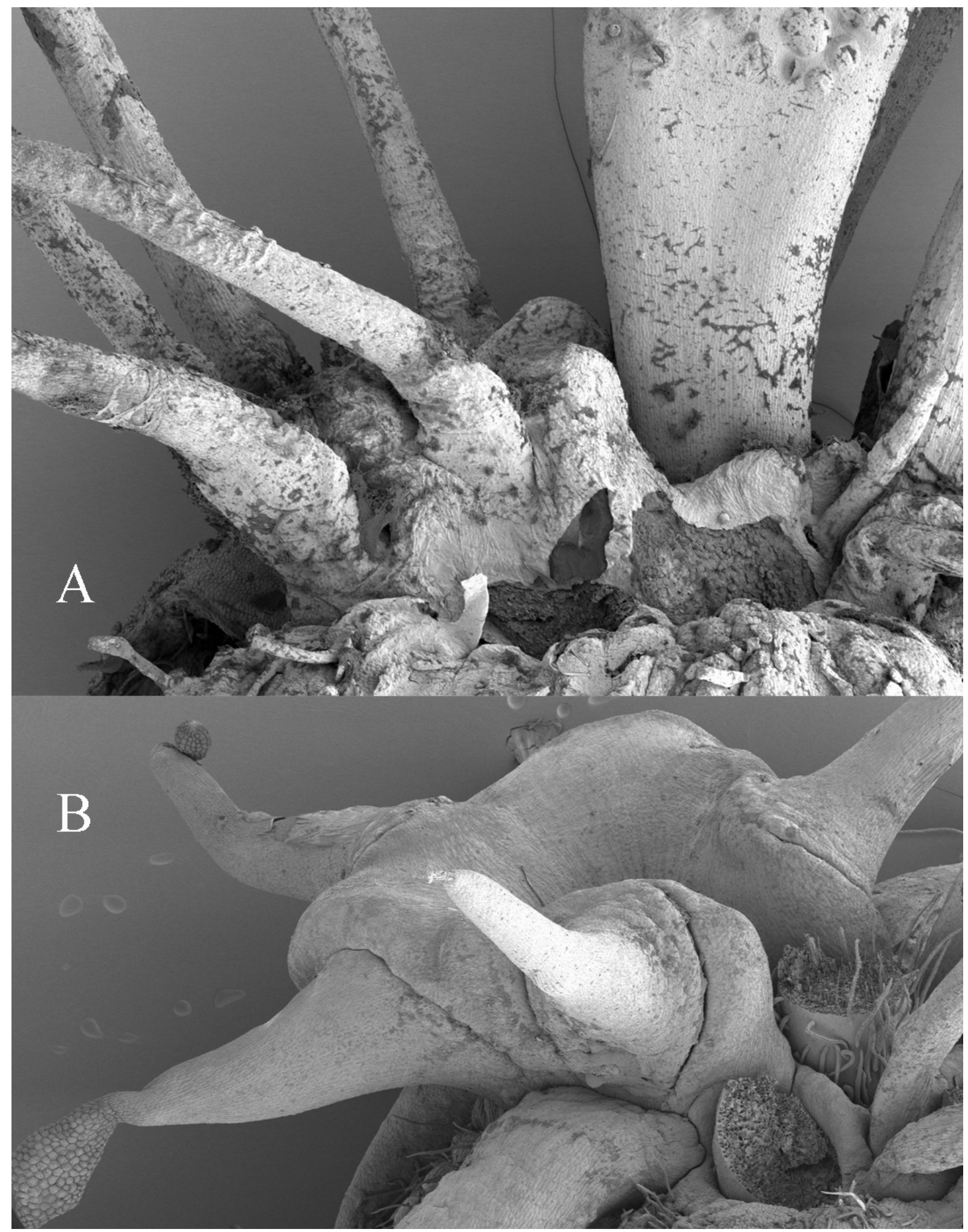

Fig. 5. Staminodes, showing 5 of the 7 per flower mounted on a raised fleshy disc. A. Cheniella corymbosa (Roxb.) R.Clark \& Mackinder comb. nov. $(\times 30$, Nichols 1292). B. C. tenuiflora (Watt ex C.B.Clarke) R.Clark \& Mackinder comb. nov. $(\times 25$, Clark 418). 


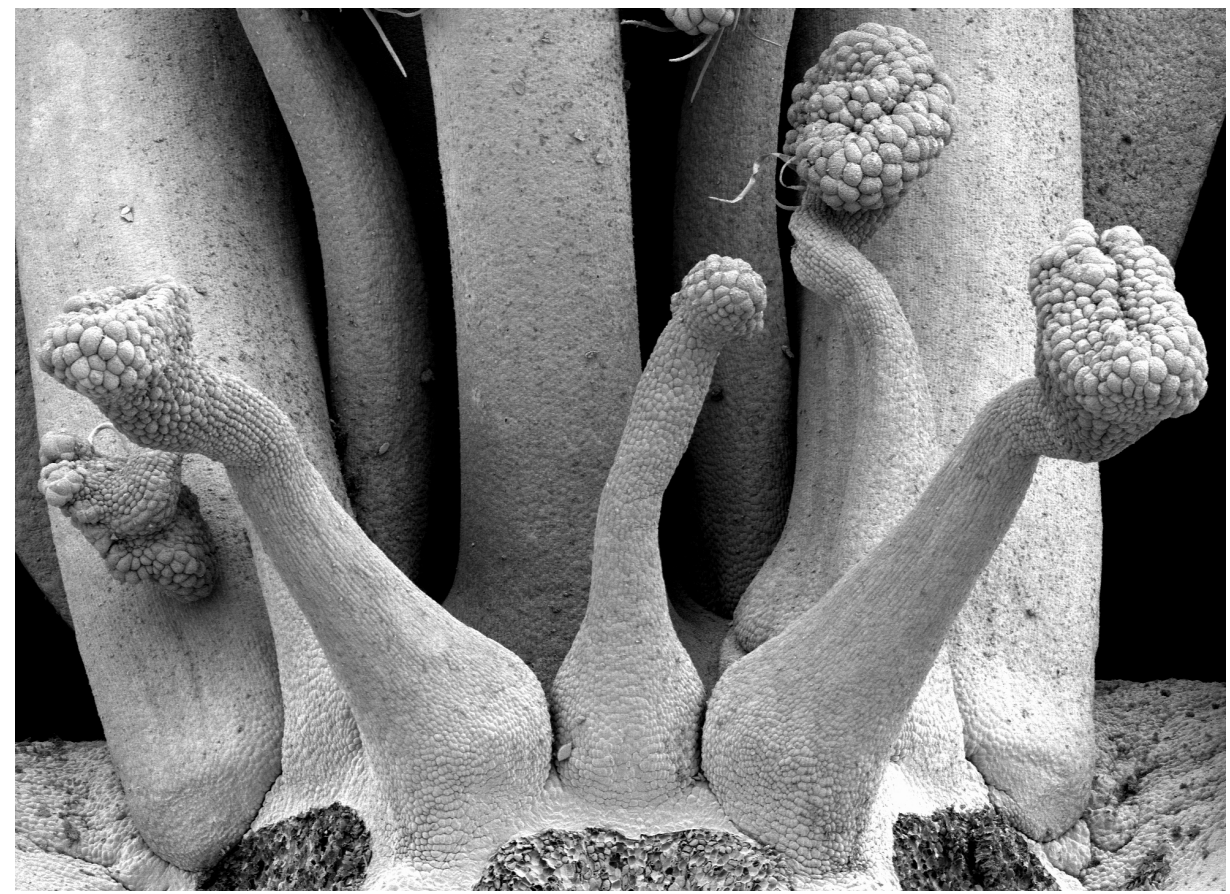

Fig. 6. Staminodes of Phanera yunnanensis (Franch.) Wunderlin, not joined at the base and lacking a fleshy disc $(\times 35$, Owens s.n. $)$.

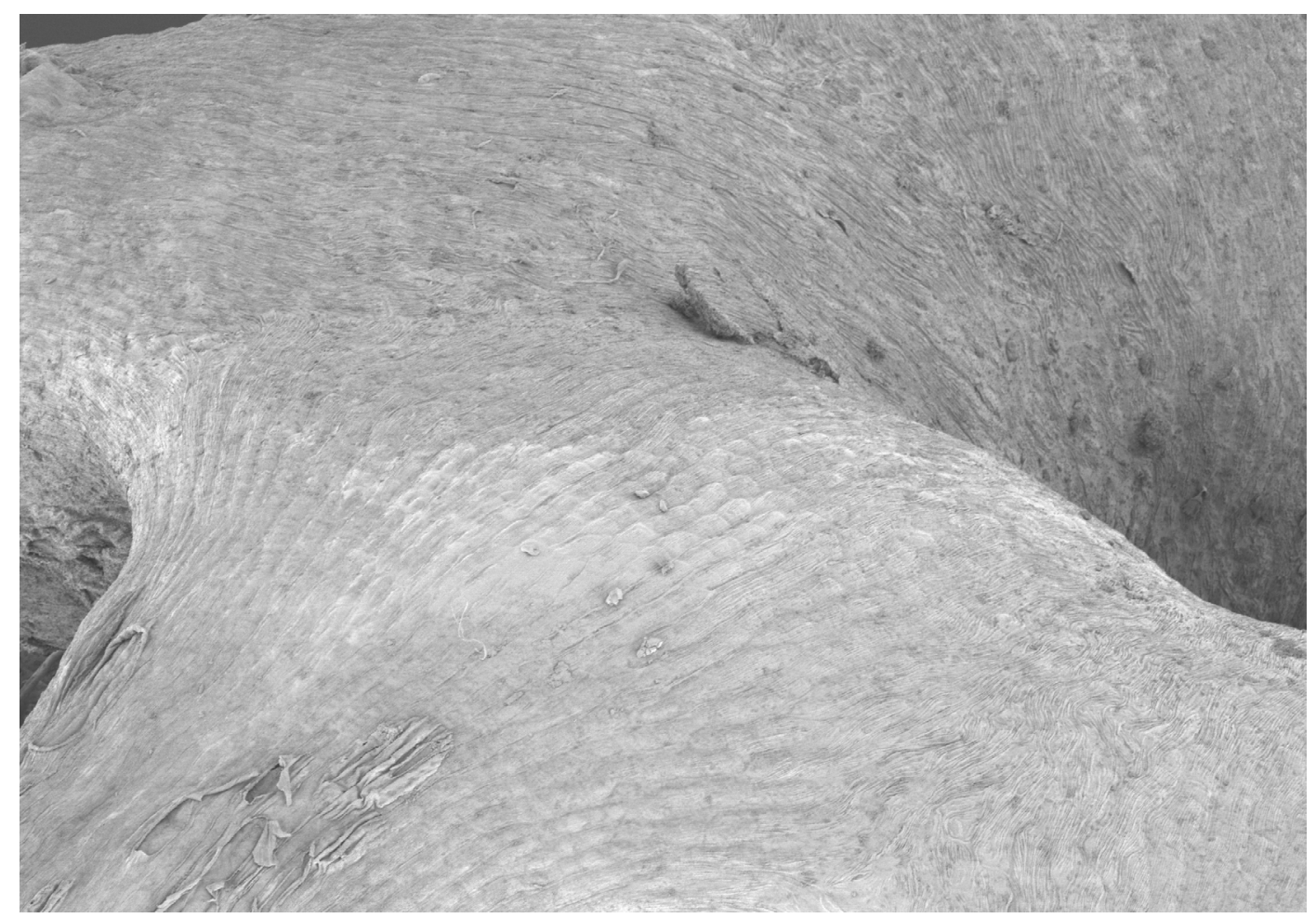

Fig. 7. Surface of staminode of Cheniella tenuiflora (Watt ex C.B.Clarke) R.Clark \& Mackinder comb. nov. $(\times 100$, Clark 426). 


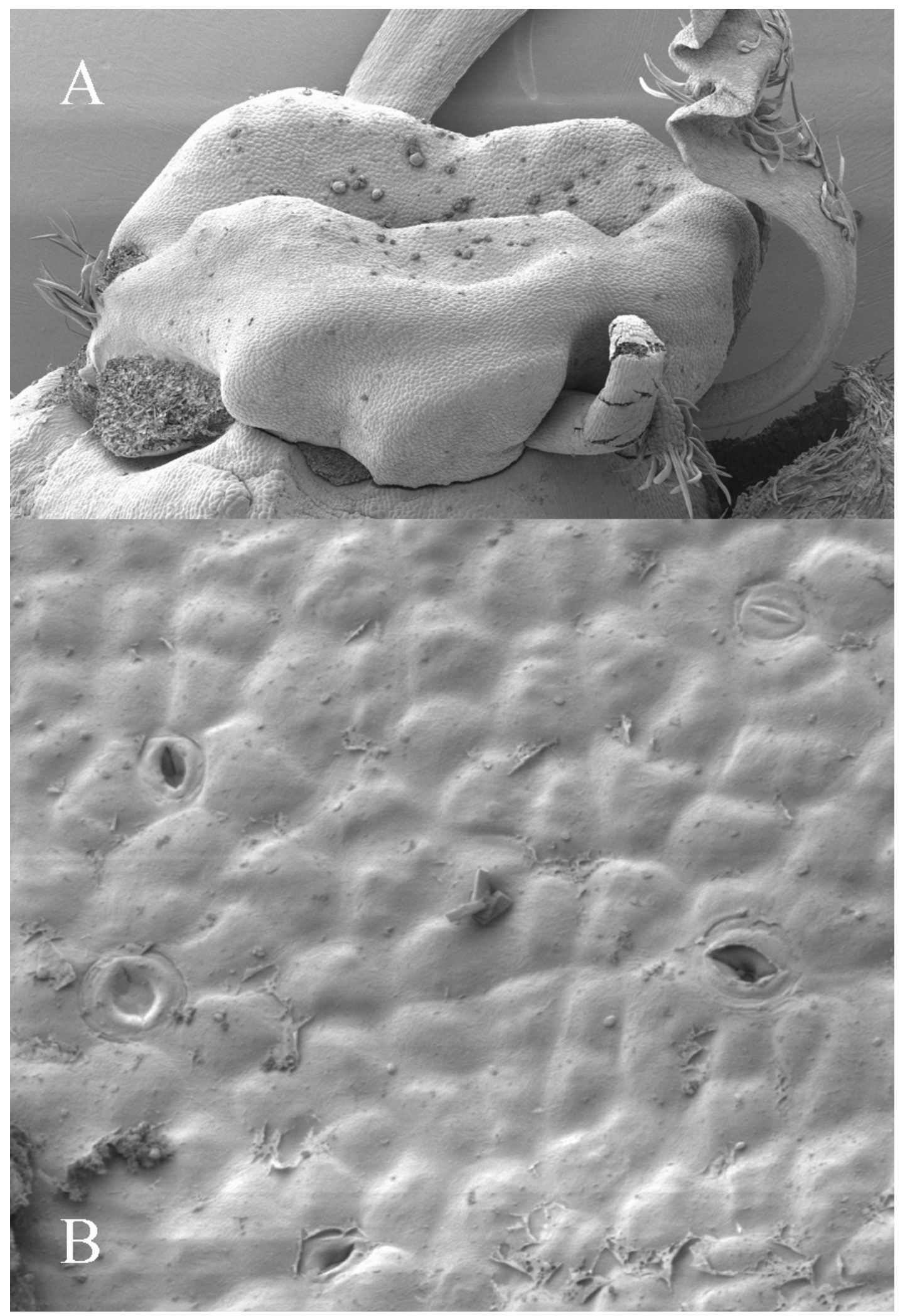

Fig. 8. Nectariferous disc of Phanera championii Benth. (Clark 458). A. Whole disc $\times 30$. B. Surface apertures $\times 450$. 


\section{Specialised morphology of Cheniella gen. nov. (2): Staminodes}

A further noteable floral morphological feature of Cheniella gen. nov. is the unusual arrangement of the staminodes. There are seven of these per flower, two of which are inserted between the stamens, with the remaining five in a separate group. The distinctive element of this arrangement within Cheniella gen. nov. is that the the group of five staminodes is inserted on a fleshy connective structure, which is raised above the hypanthium (Figs $5 \& 6$ ). This structure is not typical of the flowers of Phanera s. lat., in which the staminodes usually arise individually, directly from the hypanthium, as in the example of Phanera yunnanensis (Franch.) Wunderlin (Fig. 6).

This staminodal ring may constitute a unique synapomorphy for Cheniella gen. nov. However, possibly homologous, or at least morphologically similar structures are reported in the literature for Phanera gracillima de Wit, and have been both reported in the literature (de Wit 1956; Larsen et al. 1980; Hou et al. 1996) and observed on herbarium specimens by the present authors in Phanera japonica (Maxim.) H.Ohashi, Phanera wallichii (J.F.Macbr.) Thoth. and Phanera williamsii (F.Muell.) de Wit. We conclude that the fleshy staminodal base is indeed a synapomorphy for Cheniella gen. nov., but whether it is unique within Phanera s. lat. has yet to be confirmed.

The fleshy staminodal disc in Cheniella gen. nov. is presumed to serve some function. In a study of staminodal function and evolution, Walker-Larsen \& Harder (2000) noted that staminodes which do not serve some use within the flower probably interfere with the interactions between flowers and pollen vectors, and should therefore quickly be lost to a taxon through the processes of evolution. The reduced number of stamens and staminodes in many species of Bauhinia s. lat. indicates that a process of this

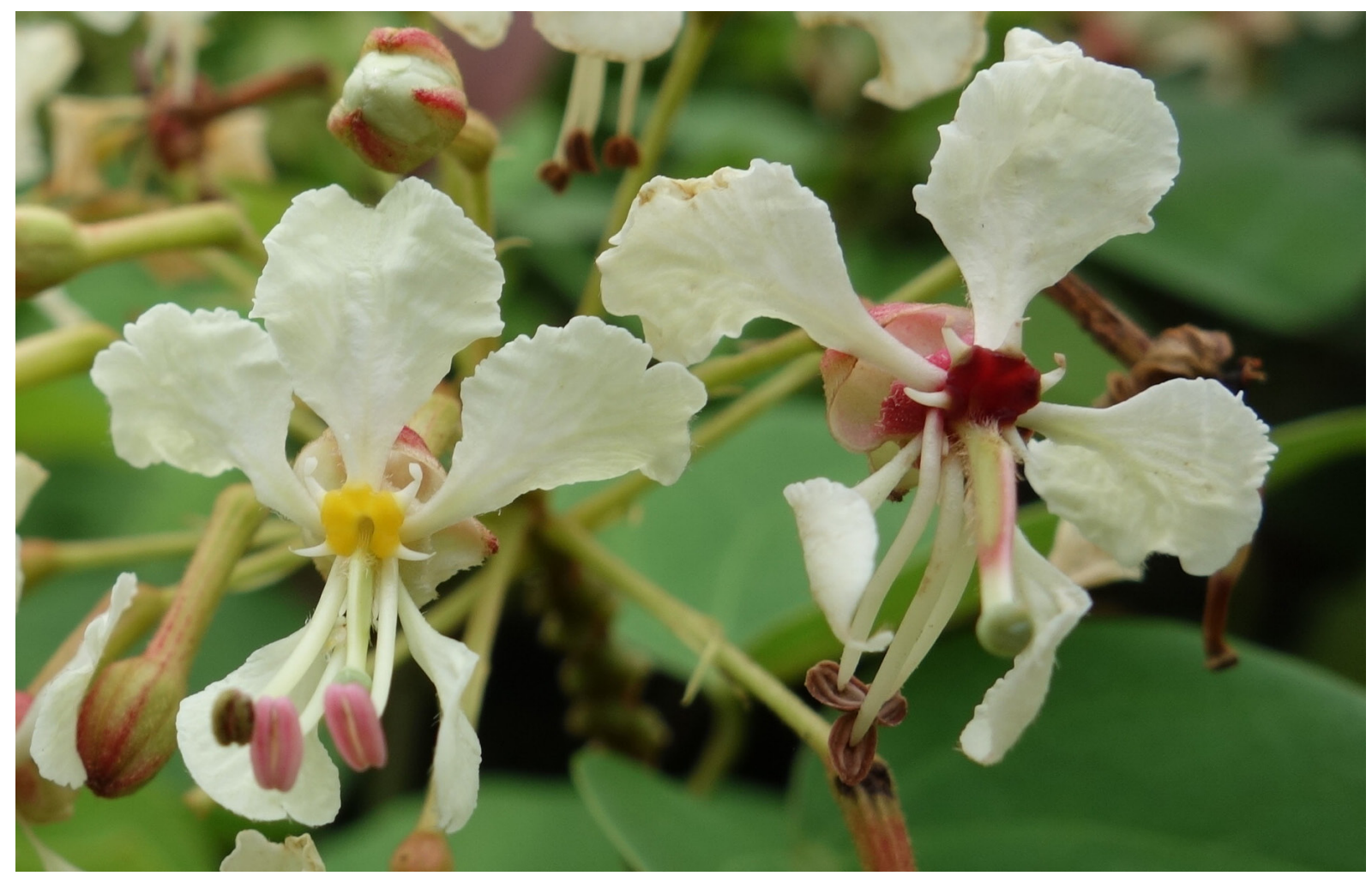

Fig. 9. Flowers of Cheniella tenuiflora (Watt ex C.B.Clarke) R.Clark \& Mackinder comb. nov., showing yellow staminodal disc in young flower and dark pink staminodal disc in old flower. Photo: R. Clark. 
nature has occurred within this group. The fact that the staminodal disc appears to be a stable characteristic within Cheniella gen. nov. therefore implies a functional role, the precise nature of which is unknown, but is most likely related to pollination. Two common staminode roles highlighted by Walker-Larsen \& Harder (2000) are pollinator attraction through the provision of attractants and rewards, and/or as a visual attractants.

Some species of Phanera s. lat., and particularly those of the potential segregate genus Lasiobema, possess floral nectariferous discs, which appear superficially similar to the staminodal disc of Cheniella gen. nov. The staminodes could be serving as a nectar secretor, as has been recorded in certain plant species such as within the Lauraceae Juss., Ochnaceae DC., Corynocarpaceae Engl., and Loasaceae Juss. (Walker-Larsen \& Harder 2000). However, SEM images of the surface of the staminodal disc in Cheniella gen. nov. show that the structure lacks apertures through which nectar might be secreted (Fig. 7), whilst distinct apertures are visible on the nectariferous discs of Phanera s. lat. (Fig. 8). The staminodal disc of Cheniella gen. nov. appears therefore not to be homologous with the nectariferous discs found within Phanera s. lat., and its potential as a nectar-producing organ is discounted.

At maturity, the staminodal disc in flowers of $C$. quinnanensis comb. nov., C. tenuiflora comb. nov. and C. touranensis comb. nov. is visually distinct, being bright yellow in contrast to the staminodes which are white, and to the petals, which are pale yellow or greenish to white (Fig. 9). When the flower is old, the colour of the disc changes to dark pink-red, or dark brown (Fig. 9). In C. corymbosa comb. nov., the staminodes are pinkish red, contrasting with the white petals (Lau et al. 2009). The distinctive colour of the staminodes in all species of Cheniella gen. nov. observed by the authors indicates a function related to pollinator attraction. This hypothesis is supported by studies on Bauhinia yunnanensis (Phanera yunnanensis) by Wang et al. (unpubl. res.), in which staminodes were experimentally removed from flowers of this species, which suggest that presence or absence of staminodes influences the composition of visiting pollinators and pollen removal and deposition.

\section{Biogeography of $C$. glauca comb. nov. and $C$. tenuiflora comb. nov.}

The decision to re-establish C. glauca subsp. tenuiflora at the species level is supported by morphological and geographical evidence. The data obtained from the specimens studied by the authors, and those seen online via the Chinese Virtual Herbarium, shows the morphological differences between C. glauca subsp. glauca and C. glauca subsp. tenuiflora to be sufficiently great to merit recognition of two distinct species. The characters that most clearly separate the two species are highlighted in bold in Table 1.

Geographically, the distributions of $C$. glauca comb. nov. and C. tenuiflora comb. nov. are almost discrete, with very little overlap between the ranges (Fig. 10). Cheniella glauca comb. nov. has a more southerly distribution, in the Thai-Malay Peninsula, extending south as far as Sumatra and Java, whereas C. tenuiflora comb. nov. occurs throughout Central and Northern Thailand, Cambodia, Laos, Vietnam, Myanmar, North-eastern India (the Seven Sister States), and Southern China. The transition between the two species occurs around the Isthmus of Kra, with a single record of C. glauca comb. nov. to the north of this region, in Myanmar. This distribution coincides with that of other plant species, many of which are confined to areas north or south of the Isthmus (Parnell 2013). The primary phytogeographical transition zone of the Thai-Malay Peninsula occurs, however, around the Kangar-Pattani Line at $6^{\circ}-7^{\circ}$ north, ca $450 \mathrm{~km}$ south of the Isthmus of Kra, where the Indochinese seasonal dry forests give way to Malesian aseasonal evergreen tropical forest (Woodruff 2010). A secondary vegetation transition zone is found ca $500 \mathrm{~km}$ north of the Kangar-Pattani Line, at Wallace's line between Mergui and Samut Songkram, where evergreen rainforest gives way to mixed moist deciduous forest (Hughes et al. 2003; Woodruff 2010; Parnell 2013). The limits of the distributions of C. glauca comb. nov. and C. tenuiflora comb. nov. intersect around this transition zone. However, it must also be noted that $C$. glauca comb. nov. is apparently not absolutely restricted to areas of rainforest and wetter, aseasonal climate: a single 


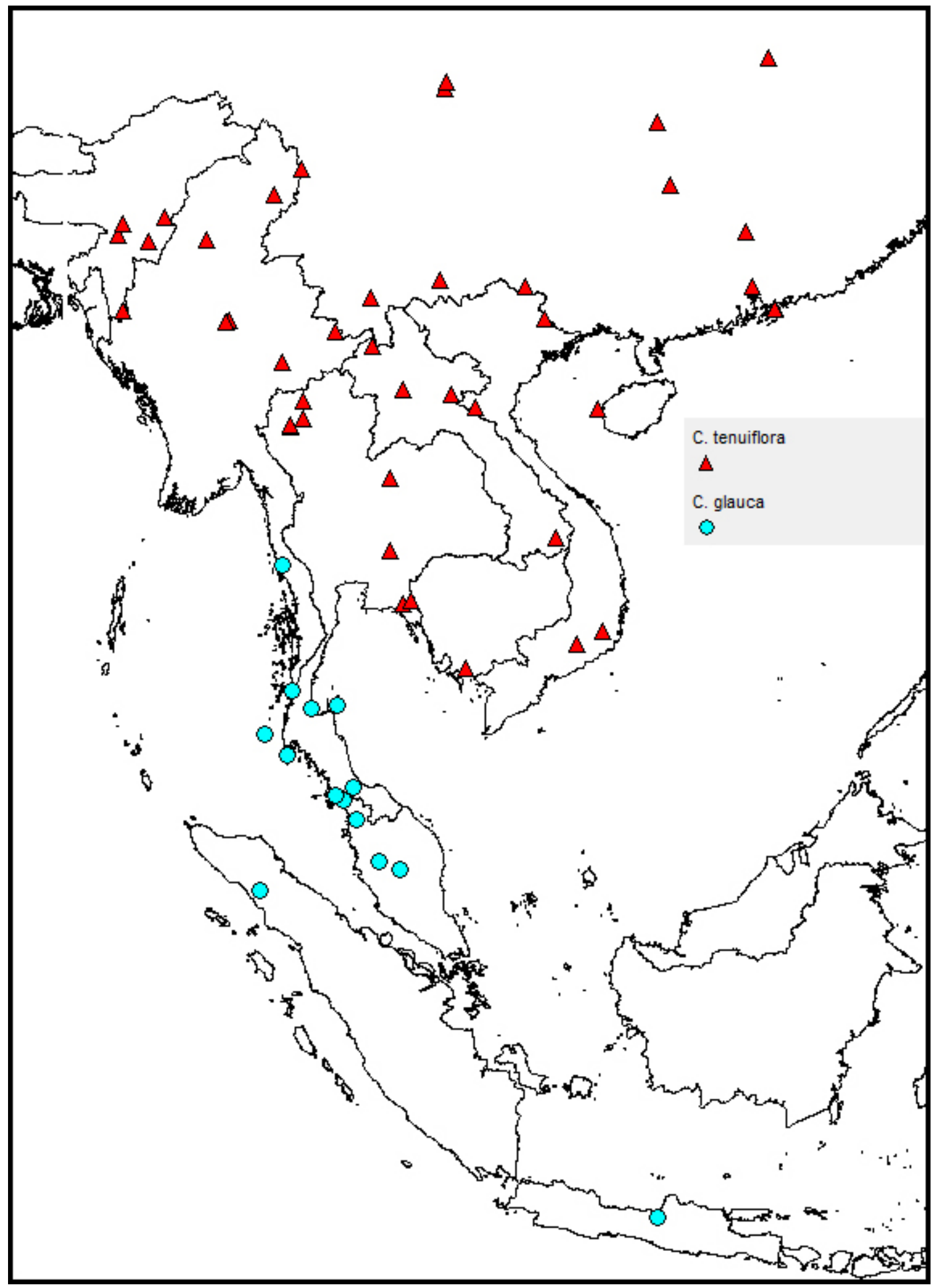

Fig. 10. Distribution of Cheniella glauca (Wall. ex Benth.) R.Clark \& Mackinder comb. nov. and C. tenuiflora (Watt ex C.B.Clarke) R.Clark \& Mackinder comb. nov. 
specimen is known from Myanmar, and one from Koi Samui, which are areas of higher seasonality (Richardson et al. 2012), and the Myanmar specimen is north of Wallace's line vegetation transition zone. The western side of the Peninsula is generally wetter than the east (Parnell 2013), which may partially explain the continuation of the range of $C$. glauca comb. nov. further north on the western side. In summary, the distributions of $C$. glauca comb. nov. and C. tenuiflora comb. nov. indicate that they have distinctly divergent habitat and climate preferences, with C. glauca comb. nov. preferring a wetter, less seasonal climate, and C. tenuiflora comb. nov. inhabiting drier areas of greater seasonality and lower rainfall. This distinction supports their recognition as separate species.

The limited molecular based phylogenetic analysis that has been carried out including C. glanca comb. nov. also provides some evidence to suggest that $C$. glauca comb. nov. and C. tenuiflora comb. nov. should not be considered as conspecific: the study of Hao et al. (2003) included one accession each of C. glauca comb. nov. (as Bauhinia glauca 1) and C. tenuiflora comb. nov. (as Bauhinia glauca 2) which were not resolved as sister lineages. Instead $C$. glauca comb. nov. was placed as sister to $C$. didyma comb. nov. albeit with low support (bootstrap $=63 \%$ ).

\section{Acknowledgements}

We are very grateful to Justin Moat and Tim Wilkinson for help in preparing the maps. Thank you to Chrissie Prychid for training and assistance in preparing the SEM samples, and to María Conejero for support with the SEM work. Many thanks to Andrew Brown for the excellent line drawing presented as Fig. 4.

\section{References}

Arroyo M.T.K. 1981. Breeding systems and pollination biology in Leguminosae. In: Polhill R.M. \& Raven P.H. (eds) Advances in Legume Systematics 2: 723-769. Royal Botanic Gardens, Kew.

Bandyopadhyay S. 2001. On the distribution range of Bauhinia glauca (Benth.) Benth. (Leguminosae: Caesalpinioideae) in India. Journal of the Bombay Natural History Society 98 (1): 149-150.

Bandyopadhyay S., Ghoshal P.P., \& Pathak M.K. 2012. Fifty new combinations in Phanera Lour. (Leguminosae: Caesalpinioideae) from Paleotropical Region. Bangladesh Journal of Plant Taxonomy 19: 55-61. https://doi.org/10.3329/bjpt.v19i1.10942

Bandyopadhyay S. \& Ghoshal P.P. 2014. Two new combinations in Lysiphyllum (LeguminosaeCaesalpinioideae). Phytotaxa 178 (4): 298-300. https://doi.org/10.11646/phytotaxa.178.4.3

Bandyopadhyay S. \& Sharma B.D. 1992. Lectotypification of Bauhinia glauca ssp. tenuiflora (Leguminosae). Journal of the Bombay Natural History Society 89 (1): 393-395.

Banks H. \& Klitgaard B.B. 2000. Palynological contributions to the systematics of detarioid legumes (Leguminosae: Caesalpinioideae). In: Herendeen P.S. \& Bruneau A. (eds) Advances in Legume Systematics 9: 79-106. Royal Botanic Gardens, Kew.

Banks H. \& Rudall P. 2016. Pollen structure and function in caesalpinioid legumes. American Journal of Botany 103: 423-436. https://doi.org/10.3732/ajb.1500248

Banks H., Feist-Burkhart S. \& Klitgaard B. 2006. The Unique Pollen Morphology of Duparquetia (Leguminosae: Caesalpinioideae): Developmental Evidence of Aperture Orientation Using Confocal Microscopy. Annals of Botany 98: 107-115. https://doi.org/10.1093/aob/mcl115

Banks H., Forest F. \& Lewis G. 2013. Palynological contribution to the systematics and taxonomy of Bauhinia s.1. (Leguminosae: Cercideae). South African Journal of Botany 89: 219-226. https://doi.org/10.1016/j.sajb.2013.07.028 
Banks H., Forest F. \& Lewis G. 2014. Evolution and diversity of pollen morphology in tribe Cercideae (Leguminosae). Taxon 63: 299-314. https://doi.org/10.12705/632.37

Banks H., Klitgaard B.B., Lewis G.P., Crane P.R. \& Bruneau A. 2003. Pollen and the systematics of tribes Caesalpinieae and Cassieae (Caesalpinioideae: Leguminosae). In: Klitgaard B. \& Bruneau A. Advances in Legume Systematics, Part 10. Higher Level Systematics: 95-122. Royal Botanic Gardens, Kew.

Bentham G. 1852. Leguminosae. In: Miquel F.A.W. Plantae junghuhnianae 2: 205-269.

Bruneau A., Forest F., Herendeen P.S., Klitgaard B.B. \& Lewis G.P. 2001. Phylogenetic relationships in the Caesalpinioideae (Leguminosae) as inferred from chloroplast $t r n \mathrm{~L}$ intron sequences. Systematic Botany 26: 487-514. https://www.bioone.org/doi/full/10.1043/0363-6445-26.3.487

Bruneau A., Mecure M., Lewis G.P. \& Herendeen P.S. 2008. Phylogenetic patterns and diversification in the caesalpinioid legumes. Botany 86: 697-718. https://doi.org/10.1139/B08-058

Chen H.Y. 1938. New species of Bauhinia from China. Journal of the Arnold Arboretum 19: 129-133.

Chen T.C. 1988. New taxa of the genus Bauhinia L. from China. Guihaia 8 (1): 43-51.

Clarke C.B. 1889. On the plants of Kohima and Muneypore. Journal of the Linnean Society, Botany 25 (165-107): 1-107.

Dezhao C., Chen T., Zhang D., Larsen K. \& Larsen S.S. (eds) 2010. Flora of China 10, Fabaceae: 6-21. Science Press, Beijing and Missouri Botanical Garden Press, St Louis.

Duarte-Almeida J.M., Negri G. \& Salatino A. 2004. Volatile oils in leaves of Bauhinia (Fabaceae Caesalpinioideae). Biochemical Systematics and Ecology 32: 747-753. https://doi.org/10.1016/j.bse.2004.01.003

Erdtman G. 1960. The acetolysis method. Svensk Botanisk Tidskrift 54: 561-564.

Ferguson I.K. \& Pearce K.J. 1986. Observations in the pollen morphology of the genus Bauhinia L. (Leguminosae: Caesalpinioideae) in the Neotropics. In: Blackmore S. \& Ferguson I.K. (eds) Pollen and Spores, Form and Function: 283-296. Academic Press, London.

Gagnepain F. 1912. Bauhinia nouveaux d'Extrême-Orient. Notulae Systematicae 2 (6): 168-182.

Gamerro J.C. \& Fortunato R.H. 2001. Morfología del polen de las especies argentinas de Bauhinia (Cercideae, Caesalpinioideae, Fabaceae). Annals of the Missouri Botanic Garden 88: 144-158. https://doi.org/10.2307/2666135

Gogoi R. \& Bandyopadhyay S. 2014. A new variety of Phanera glauca subsp. tenuiflora (Fabaceae: Caesalpinioideae) from India. Journal of the Botanical Research Institute of Texas 8 (1): 71-75.

Graham A. \& Barker G. 1981. Palynological and tribal classification in the Caesalpinioideae. In: Polhill R.M. \& Raven P.H. (eds) Advances in Legume Systematics 2: 801-834. Royal Botanic Gardens, Kew.

Hao G., Zhang D.X., Zhang M.Y., Guo L.X. \& Li S.J. 2003. Phylogenetics of Bauhinia subgenus Phanera (Leguminosae: Caesalpinioideae) based on ITS sequences of nuclear ribosomal DNA. Botanical Bulletin of Academia Sinica 44: 223-228.

Hind N. 2015. Compositae (Asteraceae). In: Utteridge T. \& Bramley G. (eds) The Kew Tropical Plant Families Identification Handbook, second edition: 206-207. Kew Publishing, Royal Botanic Gardens Kew, Richmond.

Hokche O. \& Ramírez N. 1990. Pollination ecology of seven species of Bauhinia L. (Leguminosae: Caesalpinioideae). Annals of the Missouri Botanical Garden 77 (3): 559-572. https://doi.org/10.2307/2399520 
Hou D., Larsen K. \& Larsen S.S. 1996. Caesalpiniaceae. In: Hou D., Larsen S.S., Laferriere J.E. \& Duyfjes B.E.E. (eds) Flora Malesiana. Series 1, 12 (2): 409-784. Leiden University, Leiden.

Hughes J.B., Round P.D. \& Woodruff D.S. 2003. The Indochinese-Sundaic faunal transition at the Isthmus of Kra: an analysis of resident forest bird species distributions. Journal of Biogeography 30: 569-580. https://doi.org/10.1046/j.1365-2699.2003.00847.x

IUCN. 2001. IUCN Red List Categories and Criteria: version 3.1. IUCN, Gland, Switzerland and Cambridge.

JSTOR Global Plants. 2016. Available from: http://plants.jstor.org/ [accessed 16 Feb. 2016].

Kumar R., Bandyopadhyay S. \& Sharma S. 2014. A new variety of Phanera glauca subsp. tenuiflora (Leguminosae: Caesalpinioideae) from India. Phytotaxa 166 (2): 155-159.

Lai M., Sceppa J., Ballenger J.A. \& Doyle J.J. 1997. Polymorphism for the presence of the rpL2 Intron in chloroplast genomes of Bauhinia (Leguminosae). Systematic Botany 22: 519-528. https://doi.org/10.2307/2419825

Larsen K. \& Larsen S.S. 1973. The genus Bauhinia in Thailand. Natural History Bulletin of the Siam Society 25 (1-2): 10.

Larsen K. \& Larsen S.S. 1982. Notes on some Asian Bauhinia. Nordic Journal of Botany 2: 329-332. https://doi.org/10.1111/j.1756-1051.1982.tb01196.x

Larsen K. \& Larsen S.S. 1991. Notes on the genus Bauhinia (Leguminosae-Caesalpinioideae) in SE Asia. Nordic Journal of Botany 11: 6: 629-634. https://doi.org/10.1111/j.1756-1051.1991.tb01275.x

Larsen K. \& Larsen S.S. 1993. New taxa \& nomenclatural combinations in Malesian Bauhinia (Leguminosae-Caesalpinioideae). Nordic Jornal of Botany 13: 6: 657-665. https://doi.org/10.1111/j.1756-1051.1993.tb00111.x

Larsen K., Larsen S.S. \& Vidal J. 1980. Légumineuses-Césalpinioïdées. In: Aubréville A. \& Leroy J.-F. (eds) Flore du Cambodge, du Laos et du Viêtnam: 1-227. Muséum National d'Histoire Naturelle, Paris.

Larsen K., Larsen S.S. \& Vidal J. 1984. Leguminosae-Caesalpinioideae. In: Smitinand T. \& Larsen K. (eds) Flora of Thailand. The Forest Herbarium, Royal Forest Department, Bangkok.

Larsen S.S. 1975. Pollen morphology of Thai species of Bauhinia (Caesalpiniaceae). Grana 14: 113131.

Lau C.P., Saunders R.M.K. \& Ramsden L. 2009. Floral biology, breeding systems and population genetic structure of three climbing Bauhinia species (Leguminosae: Caesalpinioideae) in Hong Kong, China. Journal of Tropical Ecology 25: 147-159. https://doi.org/10.1017/S0266467408005762

Lewis G.P. 1998. Caesalpinia-a Revision of the Poincianella-Erythrostemon Group: 15-17. Royal Botanic Gardens, Kew.

Lewis G. \& Forest F. 2005. Cercideae. In: Lewis G., Schrire B., Mackinder B. \& Lock M. (eds) Legumes of the World: 57-67. Royal Botanic Gardens, Kew.

Lewis G.P., Simpson B.B. \& Neff J.L. 2000. Progress in understanding the reproductive biology of the Caesalpinioideae (Leguminosae). In: Herendeen P.S. \& Bruneau A. (eds) Advances in Legume Systematics 9: 65-78. Royal Botanic Gardens, Kew.

LPWG (The Legume Phylogeny Working Group). 2013. Legume phylogeny and classification in the 21st century: progress, prospects and lessons for other species-rich clades. Taxon 62 (2): 217-247. https://doi.org/10.12705/622.8 
LPWG (The Legume Phylogeny Working Group). 2017. A new subfamily classification of the Leguminosae based on a taxonomically comprehensive phylogeny. Taxon 66 (1): 44-57. https://doi.org/10.12705/661.3

Mackinder B. \& Clark R. 2014. A synopsis of the Asian and Australasian genus Phanera Lour. (Cercideae: Caesalpinioideae: Leguminosae) including 19 new combinations. Phytotaxa 66: 49-68. https://doi.org/10.11646/phytotaxa.166.1.3

Merrill E.D. 1942. Records of Indo-Chinese Plants 3. Journal of the Arnold Arboretum 23: 156-197.

O'Toole C. \& Raw A. 1991. Bees of the World. Blandford, London.

Parnell J. 2013. The biogeography of the Isthmus of Kra region: a review. Nordic Journal of Botany 31: 1-15. https://doi.org/10.1111/j.1756-1051.2012.00121.x

Pérez-Barrales R., Arroyo J. \& Armbruster W.S. 2007. Differences in pollinator faunas may generate geographic differences in floral morphology and integration in Narcissus papyraceus (Amaryllidaceae). Oikos 116: 1904-1918. https://doi.org/10.1111/j.0030-1299.2007.15994.x

Punt W., Hoen P.P., Blackmore S., Nilsson S. \& Thomas A. Le. 2007. Glossary of pollen and spore terminology. Review of Palaeobotany and Palynology 143 (1-2): 1-81.

Queiroz L.P. de 2006. New species and new combinations in Phanera Lour. (Caesalpinioideae: Cercideae) from the Caatinga Biome. Neodiversity 1: 6-10. https://doi.org/10.13102/neod.11.2

Richardson J.E., Costion C.M. \& Muellner A.N. 2012. The Malesian floristic interchange: plant migration patterns across Wallace's Line. In: Gower D.J., Johnson K., Richardson J., Rosen B., Rüber L. \& Williams S. (eds) Biotic Evolution and Environmental Change in Southeast Asia: 138-163. Cambridge University Press, Cambridge. https://doi.org/10.1017/CBO9780511735882.008

Roxburgh W. 1814. Hortus Bengalensis. Mission Press, Serampore.

Roxburgh W. 1832. Flora indica ed. 1832. W. Thacker \& Co., Calcutta.

Salatino A., Blatt C.T.T., Santos W.A.C. dos \& Vaz A.M.S. da F. 1999. Foliar flavonoids of nine species of Bauhinia. Revista Brasileira de Botânica 22 (1): 17-20. https://doi.org/10.1590/S0100-84041999000100003

Santos F.A.R. dos, Novaes D.M. \& de Queiroz L.P. 2012. Pollen of Bauhinia L. and Phanera Lour. (Leguminosae-Caesalpinioideae) from the Brazilian Caatinga. American Journal of Plant Sciences 3: 909-920. https://doi.org/10.4236/ajps.2012.37108

Sinou C., Forest F., Lewis G.P. \& Bruneau A. 2009. The genus Bauhinia s.1. (Leguminosae): a phylogeny based on the plastid trnL-trnF region. Botany 87: 947-960. https://doi.org/10.1139/B09-065

Trethowan L.A., Clark R.P. \& Mackinder B.A. 2015. A synopsis of the neotropical genus Schnella (Cercideae: Caesalpinioideae: Leguminosae) including 12 new combinations. Phytotaxa 204 (4): 237252.

Vaz A.M.S. da F. 2010. New combinations in Phanera (Leguminosae; Cercideae) from Brazil. Rodriguésia 61 (Suppl.): S33-S40.

Verdcourt B. 1979. A Manual of New Guinea Legumes. Papua New Guinea, Botany Bulletin 11. Office of Forests, Division of Botany, Lae.

Walker-Larsen J. \& Harder L.D. 2000. The evolution of staminodes in angiosperms: patterns of stamen reduction, loss, and functional re-invention. American Journal of Botany 87 (10): 1367-1384. https://doi.org/10.2307/2656866 
Walter K.S. \& Gillett H.J. (eds) 1997. 1997 IUCN Red List of Threatened Plants. IUCN, Gland, Switzerland and Cambridge.

Wang Q., Song Z., Chen Y., Shen S. \& Li Z. 2014. Leaves and fruits of Bauhinia (Leguminosae, Caesalpinioideae, Cercideae) from the Oligocene Ningming Formation of Guangxi, South China and their biogeographic implications. BMC Evolutionary Biology 14: 1471-2148. https://doi.org/10.1186/1471-2148-14-88

Wilkin P. \& Schuiteman A. 2015. Orchidaceae. In: Utteridge T. \& Bramley G. (eds) The Kew Tropical Plant Families Identification Handbook, second edition: 36-37. Kew Publishing, Royal Botanic Gardens Kew, Richmond.

Wit H.C. de 1956. A revision of Malaysian Bauhinieae. Reinwardtia 3: 381-539.

Woodruff D.S. 2010. Biogeography and conservation in Southeast Asia: how 2.7 million years of repeated environmental fluctuations affect today's patterns and the future of the remaining refugial-phase biodiversity. Biodiversity and Conservation 19: 919-941.https://doi.org/10.1007/s10531-010-9783-3

Wunderlin R. 1976. Enumeration and typification of genera in tribe Cercideae. Rhodora 78: 750-760.

Wunderlin R. 1979. Consideration of Barklya and the subtribes of the Cercideae (Caesalpinioideae: Fabaceae). Phytologia 44: 325-327.

Wunderlin R.P. 2010. Reorganization of the Cercideae (Fabaceae: Caesalpinioideae). Phytoneuron 48: 1-5.

Wunderlin R.P. 2011. New combination in Phanera (Fabaceae). Phytoneuron 19: 1-2.

Wunderlin R., Larsen K. \& Larsen S.S. 1981. Cercideae. In: Polhill R.M. \& Raven P.H. (eds) Advances in Legume Systematics, Part 1: 107-116. Royal Botanic Gardens, Kew.

Wunderlin R., Larsen K. \& Larsen S.S. 1987. Reorganization of the Cercideae (Fabaceae: Caesalpinioideae). Biologiske Skrifter 28: 1-40.

Zhang D.X. 1994. Leaf venation of Cercideae (Leguminosae). Journal of Tropical and Subtropical Botany 2: $45-57$.

Zhang D.X. \& Chen T.C. 1992. Systematics and Biogeography of Bauhinia L. (Leguminosae) II. Cladistic Analysis of Ser. Corymbosae. Acta Botanica Austro Sinica 8: 31-35.

Manuscript received: 7 November 2016

Manuscript accepted: 19 January 2017

Published on: 20 October 2017

Topic editor: Koen Martens

Desk editor: Alejandro Quintanar

Printed versions of all papers are also deposited in the libraries of the institutes that are members of the EJT consortium: Muséum national d'Histoire naturelle, Paris, France; Botanic Garden Meise, Belgium; Royal Museum for Central Africa, Tervuren, Belgium; Natural History Museum, London, United Kingdom; Royal Belgian Institute of Natural Sciences, Brussels, Belgium; Natural History Museum of Denmark, Copenhagen, Denmark; Naturalis Biodiversity Center, Leiden, the Netherlands; Museo Nacional de Ciencias Naturales-CSIC, Madrid, Spain; Real Jardín Botánico de Madrid CSIC, Spain. 


\section{Appendix 1 \\ Specimens used for illustration and anatomical studies}

\section{Specimens from which the illustration was drawn}

Cheniella corymbosa (Roxb.) R.Clark \& Mackinder comb. nov.: Cult., Hort. Bot. Calcutta, s. coll. s.n. (fr) (K). C. glauca (Wall. ex Benth.) R.Clark \& Mackinder comb. nov.: Indonesia, Java, 1802-1818, T. Horsfield 165 (fl) (K). C. ovatifolia (T.C.Chen) R. Clark \& Mackinder comb. nov.: Vietnam, Cao Bang, Tra Linh District, Quoc Toan Municipality, Thang Heng (Lakes District), 666 m a.s.l., $22^{\circ} 45.25^{\prime} \mathrm{N}$, $106^{\circ} 17.58^{\prime}$ E, 6 Apr. 1998, D.K. Harder et al. 4249 (fl) (K). C. quinnanensis (T.C.Chen) R.Clark \& Mackinder comb. nov.: China, Yunnan, G. Forrest 11844 (fl) (K); India, Assam, Ridima, Naga Hills, 4800 ft., 18 May 1935, N.L. Bar 2676 (fl) (K). C. tenuiflora (Watt ex C.B.Clarke) R.Clark \& Mackinder comb. nov.: Hong Kong, Happy Valley, 1861, Urquhart s.n. (fl.) (K); Thailand, Lampang, Me Pak, $200 \mathrm{~m}$ a.s.l., 25 Oct. 1925, Winit 1455 (fl, fr) (K). C. touranensis (Gagnep.) R.Clark \& Mackinder comb. nov.: China, Yunnan, Red River Valley, Manpan, 1500 ft., A. Henry 10175 (fl) (K); Vietnam, Ha Tuyen, $\mathrm{Na}$ Hang District, at $\mathrm{Na}$ Hang power station and along trail to Ban Chou, $22^{\circ} 21^{\prime} 23^{\prime \prime} \mathrm{N}, 105^{\circ} 24^{\prime} 48^{\prime \prime}$ E, 90 m a.s.l., 7 Mar. 1994, D.K. Harder et al. 2387 (fl) (K).

\section{Specimens used for anatomical studies}

Cheniella corymbosa (Roxb.) R.Clark \& Mackinder comb. nov.: Durban Botanic Gardens [cult.], Natal, 24 Oct. 1991, G.R. Nichols 1292 (fl) (K). C. tenuiflora (Watt ex C.B.Clarke) R.Clark \& Mackinder comb. nov.: China, Guangxi Province, Jingxi city, Longlin town-An De town, $946 \mathrm{~m}$ a.s.1., $23.13503^{\circ} \mathrm{N}$, $106.10349^{\circ} \mathrm{E}, 12$ May 2016, R.P. Clark 418 (fl) (K); China, Guangxi Province, Longlin county, Da Hon Bao, 1,280 m a.s.1., 24.963819 N, $105.182544^{\circ} \mathrm{E}, 15$ May 2016, R.P. Clark 426 (fl) (K). Phanera championii Benth.: China, Yunnan Province, Xishuangbanna Botanic Garden, vine garden [cult.], R.P. Clark 458 (fl) (K). Phanera yunnanensis (Franch.) Wunderlin: USA, Florida, Miami, Fairchild Tropical Botanic Garden [cult.], Jul. 1986, S.J. Owens s.n. (fl) (K). 